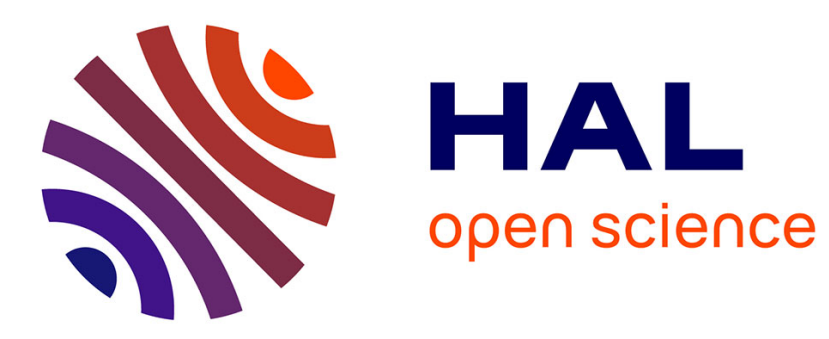

\title{
Modeling of Venus, Mars, and Titan
}

Esa Kallio, Jean-Yves Chaufray, Ronan Modolo, Darci Snowden, Robert

Winglee

\section{To cite this version:}

Esa Kallio, Jean-Yves Chaufray, Ronan Modolo, Darci Snowden, Robert Winglee. Modeling of Venus, Mars, and Titan. Space Science Reviews, 2011, 162 (1-4), pp.267-307. 10.1007/s11214-011-9814-8 . hal-00631053

\section{HAL Id: hal-00631053 https://hal.science/hal-00631053}

Submitted on 16 Nov 2016

HAL is a multi-disciplinary open access archive for the deposit and dissemination of scientific research documents, whether they are published or not. The documents may come from teaching and research institutions in France or abroad, or from public or private research centers.
L'archive ouverte pluridisciplinaire HAL, est destinée au dépôt et à la diffusion de documents scientifiques de niveau recherche, publiés ou non, émanant des établissements d'enseignement et de recherche français ou étrangers, des laboratoires publics ou privés. 


\title{
Modeling of Venus, Mars, and Titan
}

\author{
Esa Kallio · Jean-Yves Chaufray • Ronan Modolo • \\ Darci Snowden • Robert Winglee
}

Received: 9 February 2011 / Accepted: 19 July 2011 / Published online: 6 October 2011

(C) The Author(s) 2011. This article is published with open access at Springerlink.com

\begin{abstract}
Increased computer capacity has made it possible to model the global plasma and neutral dynamics near Venus, Mars and Saturn's moon Titan. The plasma interactions at Venus, Mars, and Titan are similar because each possess a substantial atmosphere but lacks a global internally generated magnetic field. In this article three self-consistent plasma models are described: the magnetohydrodynamic (MHD) model, the hybrid model and the fully kinetic plasma model. Chamberlain and Monte Carlo models of the Martian exosphere
\end{abstract}

E. Kallio (凶)

Finnish Meteorological Institute, Helsinki, Finland

e-mail: esa.kallio@fmi.fi

J.-Y. Chaufray

Laboratoire de Météorologie Dynamique, Institut Pierre Simon Laplace, Centre National de la Recherche Scientifique, Paris, France

e-mail: jyclmd@1md.jussieu.fr

R. Modolo

Université de Versailles Saint-Quentin, 45 avenue des Etats-Unis, 78035 Versailles cedex, France

e-mail: ronan.modolo@latmos.ipsl.fr

R. Modolo

Laboratoire Atmosphères, Milieux et Observations Spatiales, Quartier des Garennes, 11 bd d'Alembert, 78280 Guyancourt, France

\section{R. Modolo}

Centre National de la Recherche Scientifique, Quartier des Garennes, 11 bd d'Alembert, 78280

Guyancourt, France

D. Snowden

Lunar and Planetary Laboratory, University of Arizona, Tucson, AZ, USA

e-mail: dsnowden@u.washington.edu

R. Winglee

Department of Earth and Space Sciences, University of Washington, Johnson Hall, Box 351310, Seattle, WA 98195-1310, USA

e-mail: winglee@ess.washington.edu 
are also described. In particular, we describe the pros and cons of each model approach. Results from simulations are presented to demonstrate the ability of the models to capture the known plasma and neutral dynamics near the three objects.

Keywords Numerical modeling · Full kinetic model $\cdot$ Hybrid model · Magnetohydrodynamic model · Exosphere model · Venus · Mars · Titan · Planetary magnetospheres $\cdot$ Planetary exospheres

\section{Introduction}

Numerical simulations are commonly used to study ionized and neutral particles near Venus, Mars and Titan (VMT) because they provide a simple description of plasma phenomena and cover a wide range of temporal and spatial scales. Simulating all of the physical processes that take place in the Solar System with a single model is currently not possible. For this reason, several types of models have been developed. Each model includes approximations depending on the physical phenomena being studied. These approximations must be understood in order to interpret simulation results correctly.

In this paper we first describe three self-consistent plasma models that have been used to model the plasma and neutral dynamics in the atmospheres of VMT: (1) the magnetohydrodynamic (MHD) model, (2) the hybrid model, and (3) the fully kinetic model. MHD models simulate the dynamics of both the ions and electrons as fluids, hybrid models simulate the dynamics of ion particles and electron fluids, and fully kinetic models simulate the dynamics of ions and electrons as individual particles. Each model is used to study the plasma interaction at a different spatial and temporal scale. Roughly speaking, MHD models are used to study relatively slow, large-scale fluid processes while fully kinetic models are employed to study fast, small-scale particle processes, with hybrid models falling in between fully kinetic and MHD methods. Results are presented to demonstrate the basic phenomena simulated by each type of model. It is important to note that numerical simulations are run in discrete space and time. Therefore, a model cannot include the effects of physical processes that are not resolved spatially or temporally by the simulation, even when the processes are explicitly expressed in the model. Therefore, the spatial and temporal scales of relevant physical processes, such as the inertial lengths, gyroradius, and plasma frequencies must be considered in relation to the grid size and time step of the model.

\section{Self-consistent Plasma Modeling Methods}

In this section, we introduce MHD and hybrid models, which are three-dimensional (3D) numerical methods frequently used to analyze the plasma interactions near VMT. We also introduce fully kinetic models, which simulate positively charged ions and electrons as particles. Although, fully kinetic models have not been used to simulate the global interaction near VMT, they are introduced for theoretical completeness. Another model not discussed in depth but may be of interest to the reader is the Vlasov model. In this model each species, $s$, is described by a velocity distribution function $f_{s}(\mathbf{x}, \mathbf{v}, t)$. Interested readers can find descriptions of the Vlasov model and its usage in planetary atmospheres/ionospheres (e.g. Schunk and Nagy 2009) as well as recent reviews of MHD and hybrid modeling approaches (Ma et al. 2008; Ledvina et al. 2008) from the literature. 


\subsection{Magnetohydrodynamic Methods}

Magnetohydrodynamic (MHD) models are important tools for studying the plasma interactions of Venus, Mars, and Titan (VMT). In this section we briefly review the methods of several fluid models. Ledvina et al. (2008) is a more comprehensive review of MHD modeling methods, assumptions, and limitations. We start with ideal MHD, which is the basis for all MHD models but is rarely used to study the plasma interactions of VMT today. Next we describe multi-species MHD models, which include important mass loading and ionneutral friction terms. Hall MHD models simulate the electrodynamics more accurately by including a Hall term in the electric field equation. Multi-fluid models include the Hall term, differentiate light and heavy ion dynamics, and can include the same source and loss terms as multi-species MHD. Finally, results from various three-dimensional simulations of the plasma interaction at VMT are discussed.

The plasma interactions of VMT are described in detail in Bertucci et al. (2011, this issue). In summary, the solar wind or magnetospheric plasma and magnetic field piles-up upstream of VMT. The magnetic field drapes around the body and solar wind (or magnetospheric) plasma and field is diverted around a cavity called the induced magnetosphere. For Venus and Mars the very outer boundary of the interaction is the bow shock. This boundary does not form around Titan unless it exits Saturn's magnetosphere and enters the solar wind. Downstream of the bow shock, the next boundary layer is the induced magnetosphere boundary (IMB), also known as the magnetic pile-up boundary (MPB), where there is a strong increase in the magnetic field. Another lower boundary occurs when collision processes begin to dominate in the ionosphere. As described in Bertucci et al. (2011, this issue), the aspects of the lower boundary are significantly different for VMT.

Models of the plasma interaction at VMT are useful tools for understanding the threedimensional characteristics of induced magnetospheres and how they are affected by changes in the upstream conditions, the properties of the ionosphere, or, in the case of Mars, crustal magnetic fields. Simulations have also quantified the loss of ionospheric ions to better understand how the plasma interaction erodes the upper atmosphere.

\subsubsection{Assumptions of MHD Models}

Compared to fully kinetic or hybrid models, fluid models make the most assumptions, however these assumptions allow MHD models to be numerically simple enough to simulate even large global magnetospheres with good resolution with modest computational resources. While finer details of the magnetospheric interactions such as chemical reactions, charge exchange, and intrinsic crustal magnetic fields (in the case of Mars) can be included in hybrid models, MHD simulations are often the first to describe these physical interactions.

The core assumption of any MHD model is that the plasma acts like a fluid, bound together either by frequent collisions or by electromagnetic forces. A fluid model can only simulate the bulk parameters (velocity, density, temperature) of the interaction; therefore, it is assumed that kinetic processes stemming from the generation of energetic tails in the particle distributions or from temperature anisotropies are not important (at least to the processes that are being studied), and that the plasma behavior is well described by a single Maxwellian distribution in ideal MHD or by multiple Maxwellian distributions in the multifluid approach. In addition, all fluid models assume: quasi-neutrality $n_{e}=n_{i}, m_{i} / m_{e} \gg 1$ (neglect $d \mathbf{J} / d t$ ), and isotropic temperatures ( $T_{\perp} \approx T_{\|}$, relative to the magnetic field). Here $m$ and $n$ are the mass and number density and the subscripts $e$ and $i$ refer electrons and ions; $T_{\perp}$ and $T_{\|}$are the temperatures perpendicular and parallel to the magnetic field; and $\mathbf{J}$ is the current. 
There are two fundamental areas where fluid models differ: the treatment of Ohm's law and the treatment of ion dynamics. Ohm's law, which relates the bulk plasma properties to the induced electric field, can be derived from the electron momentum equation under the assumptions of quasi-neutrality and $m_{i} / m_{e} \gg 1$. Different versions of Ohm's law have been developed, depending on the relevant scale sizes of structures incorporated within the model. Ideal MHD and resistive MHD treatments neglect the differential acceleration of ions with different masses and assume all species have the same bulk velocity, i.e. $\mathbf{V}_{i}=\mathbf{V}_{e}=\mathbf{V}$. The resulting Ohm's law is given by either:

$$
\begin{aligned}
& \mathbf{E}=-\mathbf{V} \times \mathbf{B} \quad(\text { ideal MHD }) \\
& \mathbf{E}=-\mathbf{V} \times \mathbf{B}+\eta \mathbf{J} \quad(\text { ideal resistive MHD) }
\end{aligned}
$$

where $\mathbf{V}$ is the velocity vector, $\mathbf{E}$ is electric field, $\mathbf{B}$ is the magnetic field, and $\eta$ is the plasma resistivity (only included in resistive MHD models). If the system includes structures on the order of the ion gyroradius or ion skin depth then higher order corrections, specifically the Hall and $\nabla p_{e}$ terms should be included:

$$
\mathbf{E}=-\mathbf{V} \times \mathbf{B}+\frac{\mathbf{V} \times \mathbf{B}}{q n_{e}}-\frac{1}{q n_{e}} \nabla p_{e}+\eta \mathbf{J} \quad \text { (Generalized Ohm's Law) }
$$

where $p_{e}$ is the scalar electron pressure and $q$ is the elementary charge. Some treatments neglect the $\nabla p_{e}$ term if the electrons are cold. The effects of the Hall and $\nabla p_{e}$ terms are greatest when the ion skin depth $\left(c / \omega_{p i}\right.$ where $\omega_{p i}$ is the ion plasma frequency and $c$ is the speed of light) is comparable to the scale length of the structure $(L)$.

In addition, many fluid models assume that all ion species have the same bulk speed $\mathbf{V}$. This assumption means the model includes only one equation of motion for all of the ions, which greatly reduces the numerical complexity. However, the acceleration of different ion species depends on the ion mass and temperature. For example, low-energy ionospheric outflows can exhibit differential acceleration and propagation between light and heavy ions. Ideal, multi-species and Hall MHD methodologies neglect these effects. Multi-fluid models include them by incorporating a separate equation of motion for each ion species.

Of course, the various models also make fundamentally different assumptions about which magnetosphere-ionosphere interactions are important to the interaction region that they are studying. Some models include detailed chemistry and ionizations sources in the ionosphere, while others include relatively simple inner boundary conditions. The treatment of ion-neutral, ion-ion collisions, charge exchange, photoionization, and electron impact ionization are important when describing features that occur close to or below the planets exobase and ion outflow.

Some of the underlying assumptions of MHD models are invalid in regions near VMT. To determine whether an assumption is valid, the grid size of the simulations should be compared to the implicit length scale of the assumption. The simulation grid size is usually constrained by computational resources and the size of features of the plasma interaction. In the case of Earth's magnetosphere the bow shock should be included in the simulation. The bow shock is $\sim 15$ Earth radii from the center of the Earth at the sub-solar point and can flare out $\sim 100$ Earth radii at the flanks. Therefore, the volume of the simulation limits the grid size to a significant fraction of an Earth radius. Simulations of the induced magnetospheres of VMT are smaller relative to the planetary radius. The subsolar locations of the bow shocks of Venus and Mars are $\sim 1.5$ planetary radii $(R)$ from 
Table 1 Comparison of plasma relevant scale sizes at VMT and simulation grid sizes

\begin{tabular}{llll}
\hline & Venus & Mars & Titan \\
\hline Radius & $6052 \mathrm{~km}$ & $3395 \mathrm{~km}$ & $2575 \mathrm{~km}$ \\
$L=0.1 R, r_{l} / L$ & 0.063 & 0.43 & 1.6 \\
$\lambda_{D} / L$ & $9.3 \times 10^{-7}$ & $6.4 \times 10^{-6}$ & $7.4 \times 10^{-5}$ \\
$\lambda_{m f p} / L$ & $2.2 \times 10^{4}$ & $5.5 \times 10^{5}$ & $1.4 \times 10^{9}$ \\
$c / \omega_{p i} / L$ & $9.7 \times 10^{-3}$ & 0.047 & 0.79 \\
$c / \omega_{p e} / L$ & $2.2 \times 10^{-4}$ & $1.1 \times 10^{-3}$ & $3.8 \times 10^{-3}$ \\
\hline
\end{tabular}

the center of the planet with flare distances of less than 10 planetary radii. This means that simulation grid sizes can be on the order of $\sim 0.1 R$ or less. For the smaller bodies, Mars and Titan, the grid size is often smaller than the ion skin depth and boundary layers of the interaction can be resolved. For example, the thickness of the bow shock and magnetic pile-up boundary at Mars and Venus are on the order of the ion skin depth (Mazelle et al. 2004) as is the current sheet thickness at Titan and Mars (Halekas et al. 2006; Wahlund et al. 2005). However, resolving the ion skin depth violates the assumptions of some models. In Table 1, the implicit length scales of various MHD assumptions are compared to a typical scale size of simulations at VMT, $L \sim 0.1 R$. The scale sizes in Table 1 are taken from Ledvina et al. (2008) and are calculated for values applicable to the solar wind (for Mars and Venus) and for magnetospheric $\mathrm{O}^{+}$for Titan. The term in the first row of the table, $r_{l} / L$, compares the Lamour radius of ions in the induced magnetosphere to the simulation scale length. From this comparison it is evident that neglecting ion gyroradius effects of incident ions is acceptable at Venus, somewhat invalid at Mars, and completely invalid at Titan. However, it is important to note that these values are representative of the $\mathrm{H}^{+}$ions in the solar wind and not the heavy ions in each body's upper atmosphere. Even for Venus, the gyroradius of planetary ions can be large relative to the simulation scale length depending on the strength of the magnetic field and the origin of the planetary ions (see, for example, Kallio and Jarvinen 2011, Fig. 2). The large gyroradius of ions in VMT's ionospheres can lead to large asymmetries in the plasma interaction. For example, the convective electric field in the solar wind (or Saturn's magnetosphere) accelerates ions away on one side of VMT's ionosphere forming an asymmetric wake region. On the other side, the convective electric field accelerates ions towards the ionosphere, depositing energy into the upper atmosphere.

In each case comparing the Debye length to the simulation scale length, $\lambda_{D} / L \ll 1$, validates the assumption of quasi-neutrality. The comparison of the mean free paths, $\lambda_{m f p} / L$, and the simulation scale size shows that outside the dense regions of the atmospheres the plasma becomes collisionless; therefore, it is not valid to assume the plasma has a thermal distribution and isotropic pressure. The next two length scales, $c / \omega_{p i} / L$ and $c / \omega_{p e} / L$, are the characteristic length scales of waves that oscillate near the ion plasma frequency and electron plasma frequency. While it is a valid assumption to neglect all waves with frequencies on the order of the electron plasma frequency, neglecting waves on the order of the ion plasma frequency, such as ion cyclotron waves, is not valid at the typical resolutions of simulations of Mars and Titan.

Comparing the length scales of each of the fundamental assumptions indicates that MHD models of Venus break the fewest assumptions because of the large size of Venus compared to Mars and Titan and the relatively stronger magnetic field (which decreases the Lamour radius). MHD models of Titan's induced magnetosphere break the most assumptions. Not only is Titan small compared to Venus and Mars, Titan's ionosphere contains very massive 
ion species (Waite et al. 2005). Furthermore, the plasma in Saturn's magnetosphere contains ions that gyrate with a radius on the order of the diameter of Titan (Hartle et al. 2006).

In particular, assumptions that the plasma has a Maxwellian distribution and that ion cyclotron effects are not important become invalid in the near collisionless regions near VMT's atmospheres and in VMT's ion tails.

However, useful model-data comparisons have been made using fluid models when the authors were aware of how the limitations of their fluid simulation affected their results. Furthermore, there are several advantages to fluid models that make certain types of simulations more convenient to implemented MHD rather than fully kinetic or hybrid models. MHD models require less computational resources therefore fluid simulations typically have faster simulation times, larger simulation volumes, and good resolution inside the ionosphere of the target object. In addition, the most resolved regions in fluid simulations are often in regions where ion-neutral collisions validate the assumptions of neglecting the ion gyroradius and anisotropic pressure. Outside of the dense regions of the atmospheres it is better to use a Hall MHD or multi-fluid models to study the interaction because these models include ion cyclotron effects, although not as explicitly as hybrid models. Of course, Hall MHD and multi-fluid simulations also assume isotropic pressure and thermal plasma distributions. To simulate non-thermal distributions, it is necessary to use a kinetic or hybrid model.

\subsubsection{Ideal MHD}

The ideal MHD equations self-consistently solve for the evolution of the gas dynamics of the plasma (through the continuity, energy, and momentum equations) and the evolution of the magnetic field (through the induction equation). Here we assume the reader is familiar with MHD theory. For more detail the reader is referred to text such as Schunk and Nagy (2009). The basic form of the ideal MHD equations is:

$$
\begin{array}{ll}
\text { Continuity equation: } & \frac{\partial \rho}{\partial t}+\nabla \cdot \rho \mathbf{V}=0, \\
\text { Momentum equation: } & \frac{\partial \rho \mathbf{V}}{\partial t}+\nabla \cdot(\rho \mathbf{V V})=\mathbf{J} \times \mathbf{B}-\nabla p, \\
\text { Energy equation: } & \frac{\partial e}{\partial t}+\nabla \cdot(e \mathbf{V})=-p \nabla \cdot \mathbf{V}, \\
\text { Induction equation: } & \frac{\partial \mathbf{B}}{\partial t}=\nabla \times(\mathbf{V} \times \mathbf{B})
\end{array}
$$

where $\rho$ is the plasma mass density, $\mathbf{V}$ is the velocity vector, $\mathbf{J}$ is the current, $\mathbf{B}$ is the magnetic field, $e$ is internal energy density, and $p$ is the scalar thermal pressure. The thermal pressure is related to the internal energy density by $p=(\gamma-1) e$ and $\gamma$ is the adiabatic index, which is $5 / 3$ for an adiabatic flow.

The ideal MHD equations are written in conservative form, meaning that mass, pressure, and momentum is strictly conserved. However, sometimes the MHD equations used in models are not conservative but are in "primitive form". Primitive equations do not strictly conserve energy but can be easier to solve numerically. Some MHD equations such as the multifluid equations cannot be conservative. The errors resulting from using non-conservative forms of MHD equations are not well understood and we refer the reader to Ledvina et al. (2008) for a more detailed description of this issue. 


\subsubsection{Resistive MHD}

The diffusion of the magnetic field due to electron-neutral collisions in the atmospheres of VMT can be accounted for by using resistive MHD. The induction equation in resistive MHD is:

$$
\text { Induction equation: } \frac{\partial \mathbf{B}}{\partial t}=\nabla \times(\mathbf{V} \times \mathbf{B})+\eta \nabla^{2} \mathbf{B} .
$$

The final term on the right hand side is a resistive term that simulates the diffusion of the magnetic field inside the atmosphere due to electron-neutral collisions, where the resistivity is:

$$
\eta=\frac{1}{\sigma_{0} \mu_{0}}=\frac{\sum_{n=\text { neutrals }} v_{e n} m_{e}}{q^{2} n_{e} \mu_{0}} .
$$

Here $\sigma_{0}$ is the conductivity, $\mu_{0}$ is the permeability of free space, and $v_{e n}$ is the electronneutral collision frequency. Including this term is particularly important when studying the lifetime and structure of the magnetic field in the ionosphere. The resistive term can also be included in the multi-species and multi-fluid models below. Whether or not it is included varies from model to model.

\subsubsection{Multi-species MHD}

Multi-species MHD models improve ideal MHD by including the effects of mass loading and ion-neutral drag. Unlike ideal MHD, multi-species MHD models also calculate the relative abundance of ion species in each cell of the simulation, which depends on ion source and loss terms. The relative abundance of ion species is tracked by incorporating a separate continuity equation for each ion species:

$$
\frac{\partial \rho_{i}}{\partial t}+\nabla \cdot \rho_{i} \mathbf{V}=S_{i}-L_{i}
$$

where $i$ denotes different ion species, $S_{i}$ is the ion source term, and $L_{i}$ is the ion loss term. The source and loss terms can include elastic and inelastic collisions with the background neutral gas, photoionization, photoelectron ionization, electron impact ionization, and dissociative recombination. For more details about the source and loss terms the reader is referred to Schunk and Nagy (2009) or the papers referenced in this review.

After the continuity equations are solved the mass density of all species is summed, i.e. $\rho=\sum_{i=\text { ions }} \rho_{i}$. A single momentum equation forces the resulting single-fluid plasma:

$$
\frac{\partial \rho \mathbf{V}}{\partial t}+\nabla \cdot\left(\rho \mathbf{V} \mathbf{V}+p \mathbf{I}+\frac{B^{2}}{2 \mu_{0}} \mathbf{I}-\frac{1}{\mu_{0}} \mathbf{B B}\right)-\rho G=\frac{\partial M}{\partial t},
$$

where $\mathbf{I}$ is the identity matrix, and $\mathbf{G}$ is gravity. Collisions between ions and the planets neutral atmosphere transfers momentum from the plasma to the neutral gas, slowing the incident plasma. In addition, ions from the ionosphere are picked up by the interplanetary or planetary magnetic field. The added mass slows the propagation of the magnetic field near the body in a process known as mass loading. In the momentum equation the frictional effects of ion-neutral collisions and mass loading effect of photoionization, electron impact ionization, and charge exchange would be included in the term $\frac{\partial M}{\partial t}$, which varies from model to model. 
Similar to ideal MHD it is assumed that the dynamics of the plasma can be described by a single-fluid and that the electrons and all ions have the same bulk velocity. Therefore, the Hall term is not included in Ohm's law:

$$
\mathbf{E}=-\mathbf{V} \times \mathbf{B}+\eta \mathbf{J}
$$

This is the resistive form of Ohm's law. However, it is important to note that most of the multi-species MHD models that have been used to study the interaction at VMT neglect this resistive term. Ohm's law is substituted into the induction equation:

$$
\frac{\partial \mathbf{B}}{\partial t}=-\nabla \times \mathbf{E}
$$

to solve for the magnetic field.

The final equation describing the dynamics of the plasma is the energy or, as shown here, the pressure equation:

$$
\frac{1}{\gamma-1} \frac{\partial p}{\partial t}+\frac{1}{\gamma-1}(\mathbf{V} \cdot \nabla) p+\frac{1}{\gamma-1} p(\nabla \cdot \mathbf{V})=\frac{\partial E}{\partial t}
$$

The relation $p=(\gamma-1) e$ can be used to find the corresponding energy equation. On the right hand side of the equation, heating/cooling terms due to ion-neutral, ion-ion, and ionelectron collisions would be added. In this case we have simplified the equation by representing various heating and cooling terms with the transport function, $\frac{\delta E}{\delta t}$. In multi-species models the temperatures of all ion species are averaged together and typically it is assumed that the electron and ion temperatures are equal, i.e. $\sum_{i=\text { ions }} T_{i}=T_{e}=T_{p} / 2$.

\subsubsection{Hall MHD}

The Hall term modifies the magnetic field topology and produces asymmetries in the plasma flow near VMT. For the Hall term to be non-zero $v_{i} \neq v_{e}$. Since MHD models do not solve for the ion and electron velocity independently, the relative velocity of the ions and electrons, or the current, must be calculated by taking the curl of the magnetic field, $\mathbf{v}_{H}=-\frac{\mathbf{J}}{n e}=$ $-\frac{\nabla \times \mathbf{B}}{n e \mu_{o}}$. Then the electric field equation (Ohm's law) can be modified to include the Hall term,

$$
\mathbf{E}=-\mathbf{V} \times \mathbf{B}+\eta \mathbf{J}+\frac{1}{n q} \mathbf{J} \times \mathbf{B} .
$$

The set of Hall MHD equations are then (Tóth et al. 2008):

$$
\begin{aligned}
& \frac{\partial \rho}{\partial t}+\nabla \cdot \rho \mathbf{V}=S-L \\
& \frac{\partial \rho \mathbf{V}}{\partial t}+\nabla \cdot\left(\mathbf{V} \rho \mathbf{V}+\mathbf{I} p+\mathbf{I} \frac{B^{2}}{2 \mu_{0}}-\frac{1}{\mu_{0}} \mathbf{B B}\right)=\frac{\partial M}{\partial t} \\
& \left.\frac{\partial e}{\partial t}+\nabla \cdot\left(\mathbf{V}(\varepsilon+p)+\left(\mathbf{V}+\mathbf{v}_{H}\right) \cdot(\overline{\mathbf{I}})^{2}-\mathbf{B B}\right)-\mathbf{B} \times \eta \mathbf{J}\right)=\frac{\partial E}{\partial t} \\
& \frac{\partial \mathbf{B}}{\partial t}=\nabla \times\left(\left(\mathbf{V}+\mathbf{v}_{H}\right) \times \mathbf{B}-\eta \mathbf{J}\right) \\
& e=\varepsilon+\frac{\mathbf{B}^{2}}{2}=\frac{p}{\gamma-1}+\frac{\rho \mathbf{V}^{2}}{2}+\frac{\mathbf{B}^{2}}{2}
\end{aligned}
$$


where $\varepsilon$ is the hydrodynamic energy density. Including the additional term in the electric field equation decouples the ions from the magnetic field (and the electrons which are still strictly coupled to the magnetic field). Other variations of Hall MHD have also been applied to study Mars (Harnett and Winglee 2003, 2005). In this case the methodology was referred to as non-ideal MHD and the equations of this model included the ideal MHD continuity, momentum, and energy equations but similar to Hall MHD the Ohm's law included Hall and electron pressure gradient terms that needed to be scaled by $1 / 3$ for stability.

\subsubsection{Multi-fluid}

Multi-fluid models are fundamentally different from what is typically considered an MHD model because multi-fluid models solve for the dynamics of each ion species with a separate momentum and energy equation. This means that ion and electrons are not co-added to form a single-component-plasma, requiring all ion species and electrons to have the same speed. In addition, the Ohm's law used in multi-fluid models can include the Hall and electron pressure gradient $\left(\nabla p_{e}\right)$ terms.

For simplicity we show the form of the multi-fluid model used by Harnett and Winglee (2006) with the addition of source and loss terms. The continuity equation is:

$$
\frac{\partial \rho_{i}}{\partial t}+\nabla \cdot \rho_{i} \mathbf{V}_{i}=S_{i}-L_{i}
$$

The Ohm's Law is derived from the electron momentum equation, assuming the electrons are in steady-state drift motion. The Ohm's Law used in multi-fluid models can include the Hall term, the pressure gradient term, and a resistive term:

$$
\mathbf{E}=-\sum_{i} \frac{n_{i}}{n_{e}} \mathbf{V}_{i} \times \mathbf{B}+\frac{\mathbf{J} \times \mathbf{B}}{q n_{e}}-\frac{1}{q n_{e}} \nabla p_{e}+\eta \mathbf{J}
$$

A momentum and pressure equation is solved for each of the ion species, denoted by the subscript $i$ :

$$
\begin{aligned}
& \rho_{i} \frac{d \mathbf{V}_{i}}{d t}-q n_{i}\left[\left(\mathbf{V}_{i} \times \mathbf{B}\right)-\sum_{i} \frac{n_{i}}{n_{e}} \mathbf{V}_{i} \times \mathbf{B}\right]-\mathbf{J} \times \mathbf{B} \\
& \quad+\nabla\left(p_{i}+p_{e}\right)-\rho_{i} g(r)=\frac{\partial M_{i}}{\partial t} \\
& \frac{\partial p_{i}}{\partial t}+\gamma \nabla \cdot\left(p_{i} \mathbf{V}_{i}\right)-(\gamma-1) \mathbf{V}_{i} \cdot \nabla p_{i}=\frac{\partial E_{i}}{\partial t}
\end{aligned}
$$

The current is $\mathbf{J}=\mu_{o}^{-1} \nabla \times \mathbf{B}$ and the electron velocity is $\mathbf{V}_{e}=\sum_{i} \frac{n_{i}}{n_{e}} \mathbf{V}_{i}-\frac{\mathbf{J}}{e n_{e}}$.

The equations are closed with a pressure equation for the electron fluid and the induction equation:

$$
\begin{aligned}
& \frac{\partial p_{e}}{\partial t}+\gamma \nabla \cdot\left(p_{e} \mathbf{V}_{e}\right)-(\gamma-1) \mathbf{V}_{e} \cdot \nabla p_{e}=\frac{\partial E_{e}}{\partial t} \\
& \frac{\partial \mathbf{B}}{\partial t}=-\nabla \times \mathbf{E}
\end{aligned}
$$


The advantage of multi-fluid models is that they simulate mass dependent-asymmetric behavior. The asymmetry in ion outflow produced by the convective electric field in multifluid models is similar to those of hybrid models. Multi-fluid models also include the electric fields arising from pressure gradients and ion-demagnetization across boundary layers. A disadvantage compared to hybrid models is that multi-fluid models are unable to include non-Maxwellian particle distributions as well as temperature anisotropies.

Recently, Najib et al. (2011) used a different form of the multi-fluid equations to simulate the Mars interaction. The Ohm's law (Eq. (2.22)) used by Najib et al. (2011) did not include the resistive, Hall, and pressure gradient terms. Najib et al. (2011) also assumed that the electron pressure was equal to the total ion pressure and did not solve the electron pressure equation. The multi-fluid method used by Najib et al. (2011) includes source and loss terms as well as ion-neutral and ion-ion collision terms in the continuity, mass, and pressure equations. As described in Ledvina et al. (2008), there are questions related to how multi-fluid models simulate shocks. When one species shocks in a hybrid simulation waves are produced that interact with other ion particles. Although the full spectrum of waves are not simulated in multi-fluid models, the ion fluids in a multi-fluid model are still coupled through the electric field equation. However, due to this concern, Najib et al. (2011) solved for the hydrodynamic energy density, $e_{s}=\rho_{s} \mathbf{V}_{s}^{2} / 2+p_{s} /(\gamma-1)$, instead of the pressure equations (Eq. (2.24)) outside of and near where the bow shock forms, by solving:

$$
\frac{\partial e_{s}}{\partial t}+\nabla \cdot\left[\left(e_{s}+p_{s}\right) \mathbf{V}_{S}\right]-\mathbf{V}_{s}\left[\frac{n_{s} q_{s}}{n_{e} e}\left(\mathbf{J} \times \mathbf{B}-\nabla p_{e}\right)+n_{s} q_{s}\left(\mathbf{V}_{S}-\mathbf{V}_{+}\right) \times \mathbf{B}\right]=\frac{\partial E_{e}}{\partial t},
$$

where $\mathbf{V}_{+}$is the average velocity of all ion fluids. Although, this method is not strictly conservative, Glocer et al. (2009) claims it gives "roughly correct jump conditions across shocks when the magnetic energy density is small relative to the kinetic and thermal energy densities". Glocer et al. (2009) also couples the ion fluids in their multi-fluid model by approximating the two-stream instability along field lines by adding an additional friction term to the momentum equation. This term is not included in the model in Najib et al. (2011).

\subsubsection{MHD Models of Venus' Interaction with the Solar Wind}

Venus was the first planet to be studied in depth with 3D fluid models. The ideal, singlefluid MHD model by Tanaka (1993) simulated many features of induced magnetospheres that are well known today. For example, the model was used to determine how magnetic field lines and solar wind plasma pile-up upstream of Venus and how the magnetic field slowly slips around the flanks of the planet. Tanaka (1993) showed that the field lines are bent into U-shapes as they move from the day side to the night side of the ionosphere and that $\mathbf{J} \times \mathbf{B}$ force of the bent magnetic field lines accelerates plasma along the poles of Venus' ionosphere. Eventually the mass loaded, deformed field lines were shown to convect to the night-side of Venus and detach. The detachment of the U-shaped field formed a magnetotail with a neutral sheet of ionospheric plasma separating lobes of oppositely directed magnetic field lines. Therefore, Tanaka (1993) was the first to simulate the outflow of plasma in the solar wake of an un-magnetized object. These results agreed with data from the Pioneer Venus Orbiter, which had already observed a wake of plasma and draping of magnetic fields in Venus' ionosphere (e.g. Luhmann and Cravens 1991).

A single-fluid, two-species (multi-species), MHD model (Tanaka and Murawski 1997) improved on the model published in Tanaka (1993) by adding source and loss terms to the continuity equation to represent the production and loss of $\mathrm{O}^{+}$in Venus' ionosphere by 
photoionization and charge exchange collisions with $\mathrm{CO}_{2}$. The model also included gravity and ion-neutral friction in the momentum and energy equations. The simulation results were used to investigate the location of the bow shock and the MPB and the 3D flow patterns of ionospheric $\mathrm{O}^{+}$above and below Venus' ionopause. Tanaka and Murawski (1997) showed that horizontal flow due to the magnetic tension force transports plasma from the day side to the night side. The horizontal plasma flow is fastest near the terminator where strongly bent field lines occur and slows significantly on the night side. In addition, Tanaka and Murawski (1997) further described ion outflow in Venus' magnetotail. They showed that ionospheric $\mathrm{O}^{+}$lost from Venus forms a cross-shaped ion tail with features that resembled tail rays observed by the Pioneer Venus Orbiter (e.g. Brace and Kliore 1991).

The next sequence of models focused on quantifying how mass loading due to photoionization, electron impact ionization, and charge exchange affects the location of Venus' boundary layers. Observations showed that the bow shock and MPB were located further from Venus during solar maximum than solar minimum. It was hypothesized that mass loading from higher EUV ionization caused the bow shock to move out during solar maximum, however previous 3D MHD models (Murawski and Steinolfson 1996) had not been able to show this effect. However, the simulation by Murawski and Steinolfson (1996) used an unrealistic IMF direction.

Kallio et al. (1998) used an ideal MHD model with a source term included in the continuity equation to determine if mass loading from photoionization, charge exchange, and electron impact ionization could account for the bow shock distances. At the time the mass loading rate due to charge exchange and electron impact ionization was not well known so Kallio et al. (1998) simply tripled the photoionization rate of oxygen in Venus atmosphere. Kallio et al. (1998) found that the additional mass loading caused the bow shock to move outward in agreement with theory. The peak magnetic field in the magnetic pile-up region was also stronger.

Bauske et al. (1998) also used a single-fluid MHD model to simulate the effect of mass loading due to photoionization, charge exchange, and electron impact ionization on the location of the bow shock and magnetic field barrier near Venus. Kallio et al. (1998) only included a mass loading term in the continuity equation. Bauske et al. (1998) included mass loading terms in the continuity, momentum, and energy equations. They showed that charge exchange and electron impact ionization had only a small effect, however the mass loading due to photoionization significantly changed the location of the bow shock in agreement with observations. Bauske et al. (1998) saw a larger increase in the distance of the bow shock for the same mass loading rate used in the simulation of Kallio et al. (1998) indicating that mass loading terms should be included in the momentum and energy equation in addition to the continuity equation.

Similar to Kallio et al. (1998), Bauske et al. (1998) found that additional mass loading caused the peak magnetic field in the sub-solar direction to increase. Kallio et al. (1998) argued that this effect may be due to vortices that were produced on the on the wake side of Venus. However, the model by Bauske et al. (1998) did not produce these vortices. Instead, Bauske et al. (1998) reasoned that slower convection of plasma and field slipping around the obstacle due to ion-neutral drag leads to a higher magnetic field in the magnetic pile-up region.

The first resistive, multi-species model to be applied to Venus was developed by Terada et al. (2009). The multi-species MHD model included ten ion species, a comprehensive chemical scheme and ion-neutral collisions. This model is the first three-dimensional model to include an appropriate thermal conductivity term and they were able to reproduce realistic plasma temperatures in Venus' ionosphere. 
More recently, a single-fluid, multi-species semi-time dependent MHD model was used to interpret data taken during MESSENGER's flyby of Venus (Benna et al. 2009). Venus Express was observing the properties of the solar wind outside of Venus' induced magnetosphere and observed strong rotations in the magnetic field during the MESSENGER flyby. Benna et al. (2009) combined results from four simulations, ran with upstream conditions characteristic of four rotations of the IMF. The simulations showed that Venus' magnetotail responded and rotated within minutes of rotations in the IMF.

\subsubsection{MHD Models of Mars' Interaction with the Solar Wind}

The small scale of Mars relative to Venus and the reduced strength of the IMF at Mars means that the Lamour radius of solar wind ions and ionospheric ions are large compared to Mars' radius and, therefore, may have a larger impact on the features of the interaction. However, many features of the interaction between Mars and the solar wind have been correctly simulated by fluid models.

Most three-dimensional simulations of Mars' interaction with the solar wind have been used to study how the intrinsic crustal magnetic fields affect the location of boundary layers, ionospheric plasma flow, and ion loss rates. Quantifying atmospheric escape is particularly important at Mars because quantifying the loss of Mars' atmosphere through solar wind erosion helps answer the question: where did the water go? Nagy et al. (2004) provided a detailed review of Mars' interaction with the solar wind including results based on observation and numerical modeling. In this section we review some of that material and update it with results from numerical models published since 2004.

Similar to the model of Tanaka and Murawski (1997), Liu et al. (1999) developed a twospecies, MHD model of the plasma interaction at Mars, which included simple chemistry and source and loss terms in the continuity, momentum, and energy equations. This model simulated boundary layers with sub-solar distances that were in good agreement with observations by Mars Global Surveyor (Vignes et al. 2000). The model predicted an ionospheric outflow of $\mathrm{O}^{+}$equal to $2.5 \times 10^{25} \mathrm{~s}^{-1}$. Liu et al. (2001) described the results from an updated version of this model including an additional ionospheric species $\left(\mathrm{O}^{+}\right)$. This model found a similar ionospheric loss rate $\left(3.06 \times 10^{25} \mathrm{~s}^{-1}\right)$ and boundary layer locations as the previous model. Anomalous magnetic field had been observed near Mars, so Liu et al. (2001) used the model to investigate the effects of crustal magnetic fields by adding a small surface dipole. They found that an intrinsic crustal field can significantly perturb the ionosphere and the rotation of the fields may cause the large variability observed in the location of Mars' bow shock.

Ma et al. (2002) published the first of several three-dimensional, multi-species, singlefluid MHD studies of the interaction between Mars' exosphere and the solar wind. The first model included 3-ion species $\left(\mathrm{H}^{+}, \mathrm{O}^{+}\right.$, and $\left.\mathrm{O}_{2}^{+}\right)$and major chemical reactions, photoionization, electron impact ionization, and charge exchange interactions with Mars' extended neutral oxygen corona. One objective was to simulate how crustal magnetic fields affect the location of the bow shock and MPB. Unlike the dipole field approximation used in Liu et al. (2001), Ma et al. (2002) used a crustal field modeled after spacecraft measurements of Mars' crustal field. The strongest of the magnetic fields (which occur mostly in the southern hemisphere) were placed in the sub-ram direction. Ma et al. (2002) found that crustal magnetic fields did not effect the location of the bow shock but did effect the location of the MPB. The interaction between the piled-up IMF field in the MPB and the crustal magnetic fields also produced small-scale magnetic structures known as magnetocylinders, which had been observed by spacecraft (Mitchell et al. 2001). Ma et al. (2002) also described the general 
circulation of ionospheric plasma which, in agreement simulations of Venus' plasma interaction, was upward on the day side ionosphere, day to night at higher altitudes, downward on the night side below about $250 \mathrm{~km}$ altitude, and down Mars magnetotail at higher altitudes.

Ma et al. (2004) improved on the model published in Ma et al. (2002) by including a new spherical grid structure, better resolution near the inner boundary, and an additional species $\left(\mathrm{CO}_{2}^{+}\right)$. This model was used to quantify ion outflow from Mars for several orientations of crustal magnetic field and for different solar wind conditions. The fluxes reported in Ma et al. (2007) ranged from $2.7 \times 10^{23}$ to $2.4 \times 10^{24} \mathrm{~s}^{-1}$ for nominal solar wind conditions. The range of simulated outflow is in agreement with the flux measured by Mars Express, which was estimated to be $3.2 \times 10^{23} \mathrm{~s}^{-1}$ (Barabash et al. 2007). Different orientations of the crustal magnetic field caused the outflow to change by a factor of $\sim 2$ to 3 . Forcing Mars' atmosphere with strong storm like conditions lead to an order of magnitude increase in ionospheric loss rates $\left(3 \times 10^{25} \mathrm{~s}^{-1}\right)$.

Harnett and Winglee (2003) applied a single-fluid, "non-ideal” (Hall), resistive MHD model to Mars. The model was non-ideal because it included the Hall term and the electron pressure gradient term in the Ohm's law (reduced by 1/3). Including the full terms led to unstable results. Harnett and Winglee (2003) showed that mini-magnetopauses form around the crustal magnetic fields. The inclusion of Hall term and electron pressure gradient allowed for ion demagnetization to occur across the boundary layers, which induced additional electric fields that cannot be captured with ideal MHD. Harnett and Winglee (2005) used the same model to study the effect of crustal magnetic fields when the strongest magnetic fields are located on the night side and on the dawn and dusk terminators of Mars. The crustal magnetic fields were shown to cause large perturbations in the magnetotail, much larger than the magnetic structures themselves. In particular, the crustal magnetic fields were shown to lead to asymmetries, strong density depletions, and density enhancements in Mars' magnetotail that were in good agreement with measurements made by Phobos 2 spacecraft (Lundin et al. 1990).

The first 3D resistive, multi-fluid model applied to Mars was presented in Harnett and Winglee (2006). This model included three ion species $\left(\mathrm{H}^{+}\right.$(solar wind), $\mathrm{H}^{+}$(ionosphere), $\mathrm{O}^{+}$or $\mathrm{O}_{2}^{+}$(ionosphere)) each with a separate, continuity, momentum, and pressure equation. Harnett and Winglee (2006) did not include coupling chemistry or collision terms between the ion species or the neutral exosphere. For this reason the inner boundary of the model was placed well above the exobase where collisions are infrequent. However, charge exchange collisions between solar wind protons and Mars' extended neutral exosphere occur at those altitudes ionizing Mars' extended neutral corona and mass loading the plasma. Harnett and Winglee (2006) accounted for this by placing a static density of $\mathrm{O}^{+}$at the inner boundary, which replicates a continuous source of $\mathrm{O}^{+}$but unlike the model of $\mathrm{Ma}$ et al. (2004) the inclusion of $\mathrm{O}^{+}$due to charge exchange was not done in a self consistent way.

By simulating the separate dynamics of ionospheric and solar wind ions, Harnett and Winglee (2007) were able to determine how the crustal magnetic fields affected the access of solar wind protons to the surface of Mars. Similar to the findings of Brain et al. (2010), that were based on Mars Global Surveyor measurements, in regions where the magnetic field was oriented horizontal to the surface the penetration of solar wind protons was minimal, however where the field lines were radial to the surface the density and temperature of solar wind protons was enhanced. This effect was studied for several solar wind velocities and densities as well as magnetic field orientations and for one extreme storm-like event. Harnett and Winglee (2006) found that an increase in solar wind density causes a large increase in the outflow of heavy ions relative to an increase in the solar wind speed 
(resulting in identical kinetic pressures). Although, locally, the crustal magnetic fields had a large effect on the penetration and heating of solar protons, any enhanced outflow due to regions of heating were balanced by regions of cooling since the location of the strongest crustal magnetic fields did not have a large effect on the total loss rates.

Harnett and Winglee (2006) also discussed the complexity of quantifying ion loss rates from Mars. Harnett and Winglee (2006) showed that ion loss rates depend non-linearly on a multitude of factors. The Hall term in the model caused heavy ions to be accelerated in the direction of the convective electric field of the solar wind (ion pick-up). The number of ions lost through this process depends on the gyroradius of the ionospheric ions and, therefore, scales with the mass of the ions and the strength of the IMF. A stronger IMF reduces this effect because the ion gyroradius is small. However, Harnett et al. (2006) showed that a stronger IMF also causes higher loss ions rates of in the magnetotail plasma sheet.

Harnett and Winglee (2007) also published a version of the multi-fluid model of Mars that included high resolution across the day side of Mars. These higher resolution studies were able to show that crustal magnetic fields effect the MPB not just in the southern hemisphere but when the strongest crustal fields are in the sub-ram direction the MPB is higher across the entire day side. These results are in agreement with the findings of Brain et al. (2010).

Finally, Harnett (2009) used a multi-fluid model with high-resolution ( $\sim 40 \mathrm{~km})$ across Mars night side to study the development and dynamics of magnetic flux that form due to reconnection between IMF field and crustal magnetic fields. The energy spectra from a sample satellite flying through a simulated flux rope were similar to inverted-V type spectra that had been observed near Mars (Lundin et al. 2006). Harnett (2009) also showed that flux ropes significantly enhanced the ionospheric loss rate.

Recently, Najib et al. (2011) used a three-dimensional, multi-fluid model that included the same ion source and loss terms and ion-neutral and ion-ion collision terms as the multispecie MHD model of Ma et al. (2007). Unlike the model of Harnett and Winglee (2006), the model did not include an electron pressure equation, but instead set the electron pressure gradients to be equal to the total ion pressure gradients. Najib et al. (2011) found that, compared to a single-fluid simulation, the multi-fluid simulation produced asymmetric results in agreement with previous multi-fluid simulations by Harnett and Winglee (2006). Within $300 \mathrm{~km}$ altitude, the ion density and temperature calculated by the simulation was in good agreement to the Viking data, as was previous single-fluid, multi-species simulations (Ma et al. 2007). Najib et al. (2011) noted that the Hall term only contributed small differences to the ion density at altitudes lower than $300 \mathrm{~km}$. This result is expected as below $250 \mathrm{~km}$ the ionosphere is collisional and therefore the Hall effect should be minimal (Ma et al. 2007). Results Harnett and Winglee (2006) and hybrid simulations (e.g. Modolo et al. 2005) described in the subsequent section suggest that the Hall term is more important at further radial distances.

Najib et al. (2011) noted that the asymmetries simulated by multi-fluid models due to the individual Lorentz forcing of ion species are similar to the asymmetries observed in hybrid models. However, a comparison paper by Brain et al. (2010) shows that the ion outflow predicted by hybrid, multi-fluid and MHD simulations can be quite different. Brain et al. (2010) presented a comprehensive comparison of MHD, multi-fluid, and hybrid modeling techniques that have been recently used to study Mars' plasma interaction. Brain et al. (2010) showed that even with identical upstream conditions and similar inner boundary conditions various MHD, multi-fluid, and hybrid models can produce noticeably different results, and that the differences from model to model are not always easy to explain. For example, in MHD models most ions escape directly downstream in the solar wake. In the hybrid models the plasma escaped mostly from the flank of the ionosphere where ion pick-up occurs. 
The multi-fluid results had significant ion escape both in the tail and on the pick-up flank. Based on these results, Brain et al. (2010) questioned whether the large-scale downstream outflow of ions in fluid models was physically realistic at all. Not surprisingly, the different mechanisms of ion outflow and the differences in ionospheric configuration lead to large differences in the calculated ion escape rates. In general, the results of the comparison of the models suggest that hybrid models may be better at describing ion behavior on a large scale, while the fluid models may be better at describing physics that occurs near or inside the planetary atmospheres were the plasma undergoes frequent collisions.

\subsubsection{MHD Models of Titan's Interaction with Saturn's Magnetosphere}

Titan's interaction with Saturn's magnetosphere is challenging to model because Titan's upper atmosphere is home to some of the most complex chemistry in the solar system and the plasma and magnetic field characteristics in Saturn's magnetosphere can vary with time scales of tens of minutes to hours. Additionally, frozen-in magnetic field can persist within Titan's ionosphere for more than an hour (Ma et al. 2009). Furthermore, Titan also occasionally exits Saturn's magnetosphere and enters the magnetosheath or even the unshocked solar wind. Titan is also challenging to model because ion scale lengths near Titan are similar to or even exceed Titan's radius. Similar to Mars and Venus, the large gyroradius of ions in Saturn's outer magnetosphere and in Titan's ionosphere can lead to large asymmetries in the plasma interaction.

First we briefly mention several three-dimensional models used to study the interaction before Cassini. Ledvina and Cravens (1998) was first to simulate Titan's interaction with Saturn's magnetosphere with an ideal 3D MHD including a source term in the continuity equation. Kabin et al. (1999, 2000) also used an ideal 3D MHD model with mass loading terms in the continuity, momentum, and energy equations to simulate the basic features of Titan's interaction. However, the model in Kabin et al. (1999) included a dipole field (since little was known about Titan's interaction at that time).

Nagy et al. (2001) published the first multi-species, MHD model of Titan's interaction with Saturn's magnetosphere. This model considered three light ion species $\left(\mathrm{H}^{+}, \mathrm{H}_{2}^{+}\right.$, and $\mathrm{H}_{3}^{+}$), two medium $\left(\mathrm{N}^{+}, \mathrm{CH}_{5}^{+}\right)$, and two heavy $\left(\mathrm{N}_{2}^{+}\right.$and $\left.\mathrm{HCNH}^{+}\right)$ion species. The relative distribution of the species was determined by major chemical reaction based on a 2D model by Cravens et al. (1998) and the effects of mass loading and ion-neutral collisions were considered. The results of the model were compared with data from Voyager's flyby of Titan. The results of the simulation suggested that below an altitude of $\sim 1425 \mathrm{~km}$ the ionosphere was chemically controlled rather than dynamically controlled. The loss rate calculated from the model was $6.5 \times 10^{24} \mathrm{~s}^{-1}$. Since the results were in reasonable agreement with the more simple single-fluid models of Ledvina and Cravens (1998) and Kabin et al. (1999), Nagy et al. (2001) concluded that the structure of Titan's ionosphere did not have a large impact on the general features of Titan's induced magnetosphere.

Using a single-species MHD model with mass loading and ion-neutral friction, Backes et al. (2005) found good agreement between simulation results and magnetic field observations from Cassini's TA flyby and Backes et al. (2005) used the simulation to determine the main features of Titan's magnetic tail. Subsequently, Neubauer et al. (2006) compared results from the same model magnetic field to observations from Cassini's TB and T3 flybys and found similar agreement with the bulk features of the magnetometer data. However, for each model-data comparison the model could not reproduce small-scale features in the data and the exact amplitude and timing of the major features. Neubauer et al. (2006) explained that some of the failure of the model to capture the magnetic field observations could be due to the variability of Saturn's magnetosphere, which was evident 
in the data, and fossil magnetic fields within Titan's ionosphere. Fossil magnetic fields make it difficult (if not impossible) to correctly reproduce Cassini observations with any type of plasma model. However, MHD models offer some advantages over kinetic or hybrid models when simulating the affects of fossil magnetic fields. MHD models have good resolution in the ionosphere, where ion-neutral collisions make kinetic affects less important, and the numerical simplicity of MHD models means that they can be ran relatively quickly and for long periods of time, sampling a variety of changing upstream conditions.

MHD simulations of Cassini's T32 flyby showed that MHD models are able to simulate fossil fields within Titan's ionosphere. It has long been presumed that during high solar wind pressure, Titan may find itself outside of the magnetosphere when it is near noon on Saturn's day side. The T32 flyby, which took place on 13 June 2007, indicates that this is indeed possible. During the flyby, Titan was found outside of Saturn's magnetopause, draped in northwardly directed magnetic field that could only have been the interplanetary magnetic field (Bertucci et al. 2008). The magnetic field observed deep within Titan's ionosphere indicated draping of a southward field and was likely the fossil remnants Saturn's draped magnetic field (Bertucci et al. 2008). Ma et al. (2009) used a multi-species MHD model to simulate Titan crossing a boundary where the magnetic field transitioned from being southward to northward. Indeed, MHD simulations of Ma et al. (2009) were able to reproduce the fossil fields within Titan's ionosphere and showed that fossil fields remain within Titan's ionosphere for several hours.

Ma et al. (2006) also compared results from multi-species MHD simulations to data from Cassini's magnetometer, plasma spectrometer, and Langmuir Probe from the TA and TB flybys. The simulation reproduced the asymmetric electron density measured by the Langmuir Probe, indicating that the asymmetry was due to photoionization and electron impact ionization asymmetries, which were included in the model. The results show that multi-species MHD models do a good job simulating regions of Titan's ionosphere where ion-neutral collisions are significant. One aspect of the model that did not correlate well with the data was the deceleration region on the inbound trajectory. The simulation underestimated the spatial extent of Titan's disturbance of incident plasma. This disagreement is likely due to the neglect of ion gyroradius effects. The large gyroradius of ions in Saturn's magnetosphere means magnetospheric ions can impact Titan's ionosphere even if their guiding center is several thousand kilometers from Titan (Hartle et al. 2006).

Snowden et al. (2007) simulated the interaction between Titan and Saturn's magnetosphere using a resistive, multi-fluid model. The multi-fluid simulation used upstream parameters that were an average of the TA, TB, and T3 flyby observations and found good correlation with the bulk features of the magnetic field data from each of the flybys. Figure 1 shows results from this simulation. The blue and magenta surfaces are surfaces constant density (isosurfaces) of Titan's ionosphere and ion tail. The magenta isosurface shows the extent of Titan's heavy ( $28 \mathrm{amu}$ ) ion tail and the blue isosurfaces shows the extent of Titan's light (1 amu) ion tail. The isosurfaces show significant differences near Titan. Unlike, the MHD simulations the multi-fluid model was able to capture the asymmetric ion pick-up on the flanks of Titan's induced magnetosphere. Similar to the hybrid results of Modolo and Chanteur (2008) the model shows that heavy ions gyrate far from Titan on the anti-Saturn side of Titan's ionosphere, as seen in the magenta isosurface in Fig. 1. On the Saturn facing side of the ionosphere, ions are accelerated back into the atmosphere and are heated.

Not unexpectedly, given the direction of the magnetic field, the well-confined beam of heavy pick-up ions was found to be located below each of the flybys (as seen in Fig. 1). This explains why large asymmetric features were not observed in the data and why the 


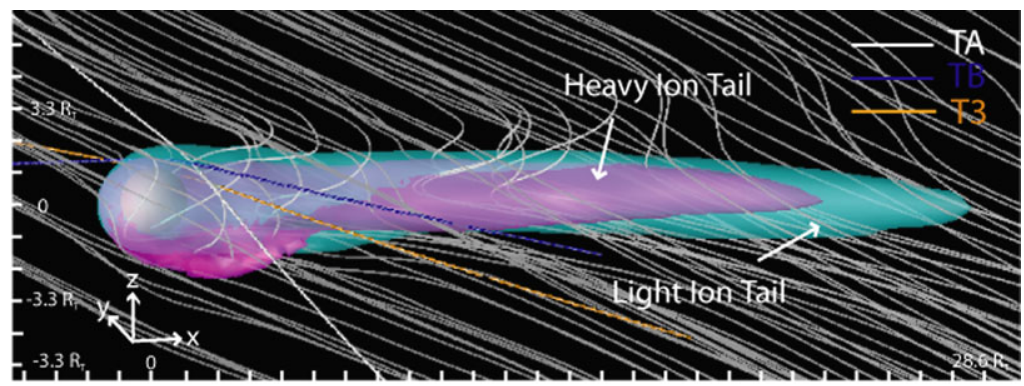

Fig. 1 Titan in Saturn's magnetosphere based on a multi-fluid model. Magnetic field lines, shown in white, interact with Titan's ionosphere. In the near Titan region the heavy ion tail (magenta) is very asymmetric. The heavy ion isosurface is drawn at a density of $10^{-1.7} \mathrm{~cm}^{-3}$ and the light ion density is shown at $10^{-1.2} \mathrm{~cm}^{-3}$ (Snowden et al. 2007)

symmetric single-fluid MHD models of Backes et al. (2005) and Ma et al. (2006) could simulate the basic features of the magnetic field data. However, the results of Snowden et al. (2007) show that asymmetric pick-up of ions on the anti-Saturn side of Titan changes the global configuration of the induced magnetosphere. The pick-up ions extend the size of Titan's ionosphere on the anti-Saturn side, causing the shielding currents to form at higher altitudes. The asymmetric pick-up also extends the mass loading and magnetic pile-up of the magnetospheric plasma and magnetic field.

Cassini's T9 flyby took place several Titan radii downstream of Titan, observing Titan's mid-wake region. The plasma instrument observed two separate ion tail regions. One region was composed of cold, heavy plasma $(\sim 16 \mathrm{amu})$. The other region was composed of warmer less massive plasma (1 and $2 \mathrm{amu}$ ). Analysis by Coates et al. (2007) and Wei et al. (2007) indicated that both regions contained plasma from Titan's ionosphere. Ma et al. (2007) simulated the T9 flyby using a Hall MHD model and a non-Hall MHD model. Ma et al. (2007) found better agreement between Cassini's magnetic field data and results from the Hall MHD simulation, indicating the importance of including the Hall term in the electric field equation. In addition, the decrease in velocity in the tail region was much better matched when the Hall term was included in the MHD model. However, the Hall MHD model still simulates all ions as a single fluid, which averages out the dynamics of ionospheric ions and ions from Saturn's magnetosphere. Therefore, the model was not able to reproduce the two compositionally distinct ion tails like the hybrid model of Modolo et al. (2007).

\subsubsection{Multi-scale Simulations of the Saturn-Titan Interaction}

The variability of Saturn's magnetosphere can strongly affect the morphology of Titan's induced magnetosphere. However, the limited scale of local models (that only simulate the region near Titan) makes realistically simulating the variability of Titan's environment difficult. To address this problem, Winglee et al. (2009) developed a multi-fluid/multi-scale model with Titan embedded within Saturn's magnetosphere. The multi-fluid aspect of the model allows the incorporation of the differential dynamics of solar wind protons, Saturn's ionospheric plasma, ions from Enceladus' plume, and Titan's ionospheric plasma. The multi-scale aspect allows Titan to be placed within a model of Saturn's magnetosphere while still maintaining sufficient resolution near Titan. 
Fig. 2 Titan embedded in a multi-fluid model of Saturn's magnetosphere. The image shows Saturn's magnetic field lines (purple) and heavy (32 amu) ion density in the orbital plane and in a plane parallel to the solar wind. Titan is placed at 21 Saturn local time and Titan's ion tail can be seen as a streak of enhanced ion density in Saturn's magnetotail

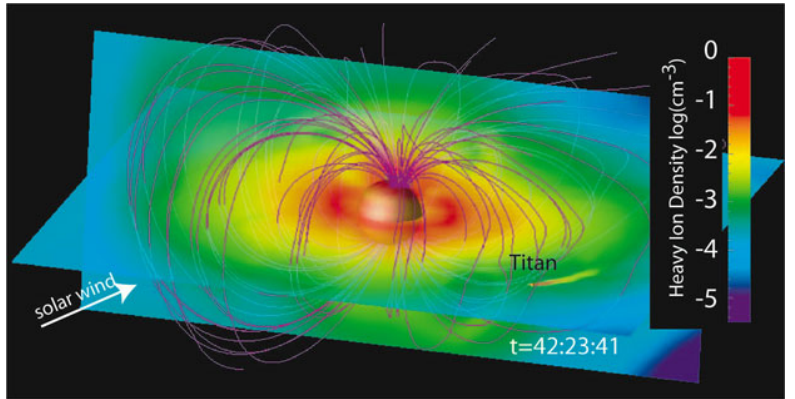

Winglee et al. (2009) examined the coupled interaction with Titan located at 21 Saturn local time. The simulation showed that Titan's induced magnetosphere is affected by the direction of the interplanetary magnetic field (IMF), because the IMF direction affects the global characteristics of Saturn's magnetosphere. Results from this simulation are shown in Fig. 2. In this figure, the heavy (32 amu) ion density is imaged in Titan's orbital plane. A streak of heavy ions can been seen downstream of Titan. The heavy ions are Titan's ion tail, which forms due to Titan's interaction with the rotating magnetic field and plasma in the Saturn simulation. Although, not shown, the finest resolution near Titan is $0.18 R_{T}$, so that the simulation can also be used to study some aspects of Titan's induced magnetosphere.

When the IMF is parallel to Saturn's planetary magnetic field, Saturn's magnetosphere near Titan was relatively stable with a thick plasma sheet. In this case, Titan's ion tail was well confined and grew to a length of over $10 R_{S}$ over several hours of simulated time. When the IMF is anti-parallel to Saturn's planetary magnetic field, the situation is more dynamic. Enhanced convection within Saturn's magnetosphere leads to an enhancement of the Rayleigh-Taylor instability between cold, dense plasma in Saturn's plasma disk and hot, tenuous plasma in the outer magnetosphere, which is driven by the outward centrifugal force of the rapidly rotating plasma. As shown by Kidder et al. (2009) and Winglee et al. (2009), the instability causes regions of cold, dense plasma to extend into Saturn's outer magnetosphere. The regions of plasma sweep past Titan and cause strong variability in the plasma and magnetic field characteristics near Titan. The variability causes Titan's ion tail to flap, impeding the formation of a complete ion torus at Titan's orbit. The outflow rate of heavy ions from Titan's ionosphere also increased from $3-4 \times 10^{24} \mathrm{~s}^{-1}$ for parallel IMF to $1.2 \times 10^{25} \mathrm{~s}^{-1}$ for anti-parallel IMF.

\subsubsection{Summary of MHD Methods}

Although, the Larmor radius of ions modeled in Venus', Mars' and Titan's interaction are significant, turbulence or wave-particle interactions may act as pseudo-collisions, particularly inside of the bow shock, reducing global asymmetries due to gyrating ions (Nagy et al. 2001). This is certainly occurring to some extent as the results of MHD simulations are in good agreement with satellite observations of the locations of boundary layers such as the bow shock and MPB. However, there are also many features of the interaction such as an extended pick-up region that cannot be simulated with a symmetric single-fluid MHD model. In fact, more groups are adopting some variation of the multi-fluid techniques to account for physics due to mass depended acceleration of ions and the Hall and electron pressure gradient term in Ohm's law. However, even these models cannot account fully for the kinetic effects of gyrating plasma, including non-Maxwellian plasma distributions and anisotropic pressure gradients, which are frequently observed near VMT. In order to account for these effects a hybrid or fully kinetic approach must be taken. 


\subsection{Hybrid Methods}

In this section, the hybrid approach is briefly detailed, emphasizing its advantages and limitations. Hybrid models are used to describe phenomena occurring at smaller ion spatial and temporal scales than simulated by MHD models, however hybrid models cannot simulate processes occurring at electron scales such as the Debye length, electron inertia length and electron cyclotron frequency. As described in the next section, a description of phenomena taking place on electron scales requires a fully kinetic model in which each species, $s$, is described by a distribution function $f_{s}(\mathbf{x}, \mathbf{v}, t)$ satisfying the Vlasov equation or when the dynamics of both ion and electron particles are simulated. However, the relatively high computational requirements needed to simulate large numbers of particles, electron length and time scales, and plasma transit times means that, currently, fully kinetic models generally cannot simulate complete magnetospheric interactions and are reserved to study local processes. Typically, the physical dimension of the simulation box is about six to ten times the radius of the obstacle in models of the plasma interaction of Venus, Mars or Titan. Fully kinetic models must resolve the electron scale (which can be of the order of few km or even less). In addition, the transit time of solar wind particles, corresponding to several hundred proton cyclotron periods, are required to reach a quasi-steady state. Therefore, to study phenomena occurring on global scales it is necessary to simplify the fully kinetic model by abandoning the description of all the detailed particle behavior.

Hybrid formalism is thus an intermediate approach between MHD (Sect. 2.1) and fully kinetic (Sect. 2.3) models. In a hybrid model, electrons are described as a mass-less chargeneutralizing fluid, whereas ions are treated as individual charged simulation particles. Simulation particles, also called macroparticles, have a volume corresponding to a simulation cell volume and their positions are identified by the center of the particle. A macroparticle can be viewed as a cloud of identical charged particles traveling together at the same velocity. Macroparticles do not have any internal degrees of freedom and cannot be considered as an elementary volume of plasma. Owing to their finite size and their ability to interpenetrate each other, macroparticles do not suffer close binary collisions. In order to represent major and minor ion species with sufficient statistical sampling, macroparticles are statistically weighted. The weight of each macroparticle determines the total particle distribution. The use of weighted macroparticles enables hybrid models to simulate regions with large density differences.

Temporal evolution of the position and speed of macroparticle number $p$ satisfies the equation of motion of individual charged particles, similar to the full particle equations:

$$
\begin{aligned}
\frac{d \mathbf{x}_{p}}{d t} & =\mathbf{v}_{p} \\
\frac{d \mathbf{v}_{p}}{d t} & =\frac{q_{p}}{m_{p}}\left(\mathbf{E}+\mathbf{v}_{p} \times \mathbf{B}\right) .
\end{aligned}
$$

Electrons are modeled by a mass-less neutralizing fluid, leading to $n_{e}=\sum_{s} n_{s}=\rho$, where $n_{e}$ is the electron number density and $n_{s}$ is the ion number density of species $s$. This assumption is valid for scale lengths larger than the Debye length, which is generally the case for plasma interactions with planetary atmospheres. The models in Simon et al. (2006) and Modolo and Chanteur (2008) used two electron fluids to represent incoming plasma and planetary plasma with different densities and temperatures while Brecht and his coworkers solve the electron energy equation for the electron temperature (see Ledvina et al. 2008, and references therein). Moments (density, velocity, ionic current, etc.) are derived from 
macroparticle information collected on a grid (Birdsall and Langdon 1985). Electric field is a function of state computed from the electron momentum equation:

$$
\mathbf{E}=-\frac{\mathbf{J}_{i} \times \mathbf{B}}{\rho}+\frac{(\nabla \times \mathbf{B}) \times \mathbf{B}}{\mu_{\rho} \rho}-\frac{\nabla p_{e}}{\rho}
$$

where $\mathbf{J}_{i}=\sum_{s} q_{s} n_{s} \mathbf{V}_{s}$ is the ionic current, $\mathbf{V}_{s}$ and $n_{s}$ are the speed and the density of ion species $s$. The total current is calculated from Ampère's equation without the current displacement term. Neglecting the current displacement term is valid as long as low frequency phenomena are of interest. Faraday's law gives the time evolution of the magnetic field, while satisfying conservation of magnetic flux:

$$
\begin{aligned}
& \frac{\partial \mathbf{B}}{\partial t}=-\nabla \times \mathbf{E} \\
& \nabla \cdot \mathbf{B}=0 .
\end{aligned}
$$

$\mathbf{E}$ and $\mathbf{B}$ can be computed on two identical grids shifted relative to each other by half a grid size in all directions allowing the simulation to resolve the conservation of the magnetic flux to the second order of approximation (Birdsall and Langdon 1985).

Time integration schemes used for the solar wind interaction with a weakly magnetized body are mainly the algorithms developed by Harned (1982), Matthews (1994) and Kallio and Janhunen (2001).

The morphology of the simulation grid varies from Cartesian grid to non-structured grid with higher spatial resolution close to the obstacle or curvilinear grid (Modolo et al. 2005; Kallio et al. 2010; Boesswetter et al. 2007).

\subsubsection{Hybrid Models of Mars' Interaction with the Solar Wind}

Since Mars does not possess a global intrinsic magnetic field, Mars' upper atmosphere and ionosphere acts as an obstacle to the solar wind flow. This property implies a strong coupling between the neutral environment, the exosphere and atmosphere, and the solar wind plasma. In addition, highly localized crustal magnetic fields have been discovered on the surface of Mars (Acuña et al. 2001), indicating that Mars possessed a strong active dynamo at some time in the past.

Several hybrid models have been adapted to investigate the solar wind interaction with the Martian atmosphere (Brecht and Ferrante 1991; Kallio and Janhunen 2001; Shimazu 1999; Boesswetter et al. 2004; Modolo et al. 2005). Each of these models has their own specifics on both numerical (simulation grid, average number of particles per cell, algorithm, etc.) and physical processes (implementation of ionization processes or ion planetary production, ionospheric chemical model, number of ion species, etc.) and they are detailed in their original article listed above. However, all of them self-consistently describe the dynamics of each ion species (solar wind/planetary ions). Since the original papers, the models have been improved either by adding a chemistry scheme (Brain et al. 2010; Brecht et al. 2010), or by improving their spatial resolution (Kallio et al. 2010), or including crustal fields (Brecht et al. 2011).

Although, Mars' crustal magnetic fields have not been incorporated into most hybrid models, mainly due to the model's limited spatial resolution, the models reproduce the most distinct plasma regions and boundaries observed near Mars with respect to in-situ observations (Figs. 3(a)-(d)). For example, the bow shock can be clearly identified in Figs. 3(a) 
a)

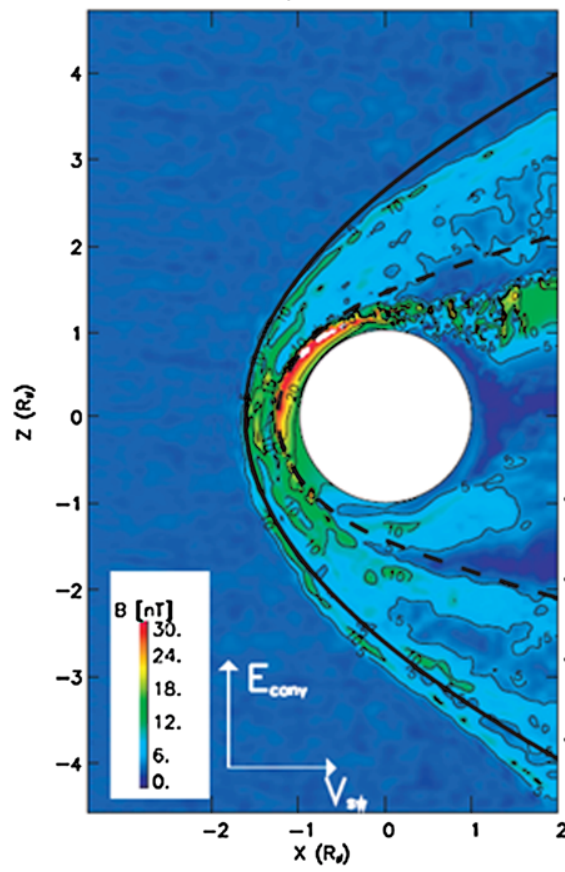

C)

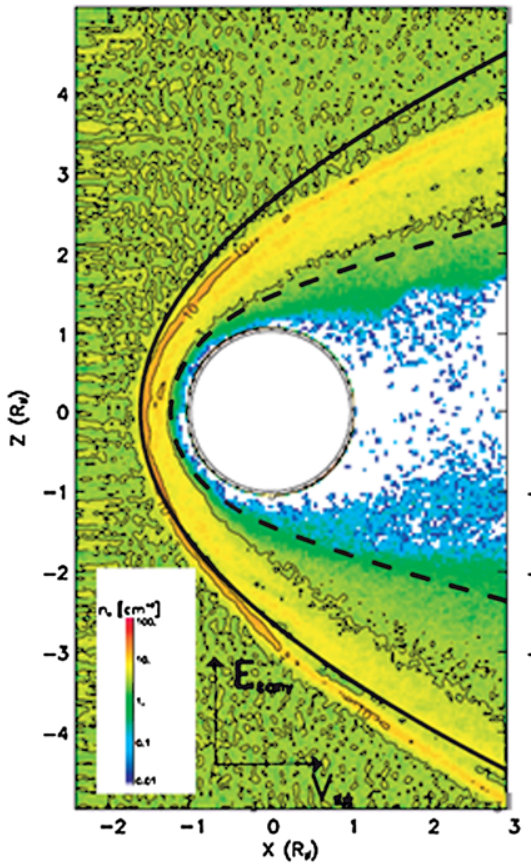

b)

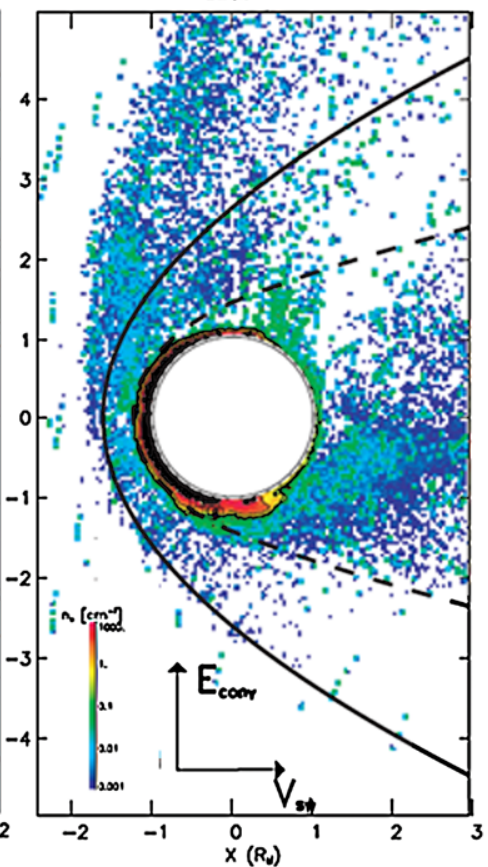

d)

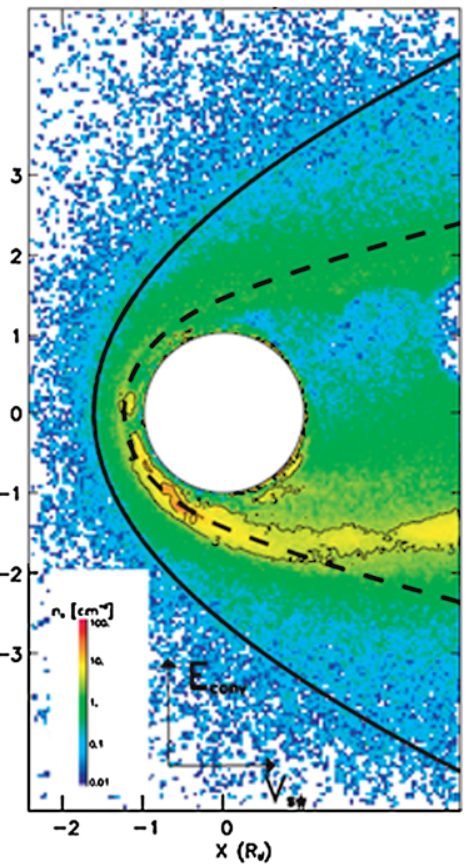

Fig. 3 Maps of total magnetic field (a) in the plane containing the motional electric field $E_{\text {conv }}$ and the solar wind flow direction $V_{s w}$. Panels (b)-(d) represent density maps of $\mathrm{O}^{+}$ions, solar wind protons and planetary protons respectively, in the same plane than panel (a). Simulated results extracted from the SWIM challenge (Brain et al. 2010) by Modolo et al. (2005) 
and 3(c). At the bow shock, the increase in magnetic field strength (Fig. 3(a)) coincides with an increase in solar wind proton density (Fig. 3(c)) and a decrease in the bulk plasma velocity (not shown). The position and shape of the bow shock are in good agreement with the average location of the bow shock deduced from Mars Global Surveyor observations, which are indicated by a black full line (Vignes et al. 2000). Multiple shock structures are usually present in hybrid simulations and are often associated with kinetic effects and multiple ion species. In addition, closer to the planet, a region of strong total magnetic field, reaching about $30 \mathrm{nT}$, is also shown (Fig. 3(a)) and corresponds to the Magnetic Pile-up Boundary (Bertucci et al. 2003) or Induced Magnetospheric Boundary (Lundin et al. 2004). The so-called Ion Composition Boundary (ICB), instead, indicates the transition from a region governed by solar wind plasma (Fig. 3(c)) to a region mainly populated by planetary plasma consisting of $\mathrm{O}^{+}$and $\mathrm{H}^{+}$ions (Figs. 3(b) and 3(d)). Hybrid models also simulate the deposition of energy into the Martian atmosphere by precipitating solar wind plasma (Brecht 1997; Kallio and Janhunen 2001; Boesswetter et al. 2004; Modolo et al. 2005) as is evident in Mars Express observations (Lundin et al. 2004; Dubinin et al. 2006).

One common feature seen in hybrid simulations is asymmetric boundary layers. The symmetry in the system is broken by the Hall term, which is included consistently in the hybrid approach. The asymmetry of the induced magnetospheric boundary is particularly apparent in the $+\mathbf{E}_{\text {conv }}$ hemisphere, in agreement with Vennerstrom et al. (2003). The density map of $\mathrm{O}^{+}$ions (Fig. 3(b)) clearly shows the asymmetric forcing of the convective electric field. In the $-\mathbf{E}_{\text {conv }}$ hemisphere the electric field points radially inward, towards Mars, while in the $+\mathbf{E}_{\text {conv }}$ the electric field points outward. The acceleration of ionospheric plasma by $\mathbf{E}_{\text {conv }}$ produces a heavy plasma plume along the $+\mathbf{E}_{\text {conv }}$ direction. Magnetic field curvature and gradients, which are due to the draping of magnetic field lines around the planet, exert magnetic tension and pressure which forces the solar wind and planetary plasma tail-ward (Figs. 3(b) and 3(d)). The different dynamics of planetary $\mathrm{O}^{+}$and planetary $\mathrm{H}^{+}$seen in Fig. 3 illustrates the necessity of modeling multiple ion species. For example, planetary $\mathrm{O}^{+}$ ions in Mars' ion tail are mainly concentrated in the plasma sheet while planetary $\mathrm{H}^{+}$are concentrated in the lobe regions. Closer to the planet, where the ionosphere is in chemical equilibrium, the plasma is more fluid-like and MHD or multi-fluid models can be more advantageous than kinetic models because they are computationally simpler and can more finely resolve the ionosphere. However, even in the ionosphere, hybrid models reproduce the global density gradients observed by Mars Express (Duru et al. 2008, 2010). In many ways, such models are in good agreement with spacecraft observations (e.g. Kallio et al. 2006; Modolo et al. 2006; Boesswetter et al. 2007; Dubinin et al. 2008).

Although hybrid models are in good agreement with recent Martian observations, significant improvements can still be made. For example, hybrid models can be improved by including a better description of the induced magnetospheric region, self-consistent ionospheric chemistry, remnant surface magnetic fields and improved coupling between charged and neutral species. A first attempt to use a 3D exospheric model coupled to a 3D hybrid model have been performed by Chaufray et al. (2007), as described in Sect. 3. The results of Chaufray et al. (2007) emphasize the need to include multiple species and kinetic effects to properly account for the plasma dynamics of each species. However, the results also illustrated how the low spatial resolution of hybrid models makes it difficult to properly include the coupling between the neutral atmosphere and the plasma. Including realistic coupling between the neutral plasma and the neutral atmosphere requires a spatial resolution lower or equal to the neutral and ionospheric scale height, which is of the order of $25-45 \mathrm{~km}$. Such spatial resolution is not yet accessible for sequential hybrid models due to computational limitations. A parallel hybrid model is needed. 


\subsubsection{Hybrid Models of Titan's Interaction with Saturn's Magnetosphere}

In the last ten years, most of the hybrid models developed to study the Mars' plasma interaction have been adapted to investigate the interaction between Saturn's magnetospheric and Titan (Brecht et al. 2000; Kallio et al. 2004; Sillanpää et al. 2007; Simon et al. 2007, 2009; Modolo and Chanteur 2008; Ledvina et al. 2011). MHD and multi-fluid models of Titan's plasma interaction have been somewhat advantageous in the dense regions of Titan's ionosphere since some models include chemical reaction and thus have a more complete description of this region (e.g. Ma et al. 2006). However, more recently, hybrid models have been developed that include a similar set of ionospheric chemistry equations (Ledvina et al. 2011). MHD and multi-fluid models can not take into account finite ion gyroradius effects. These effects cannot be ignored in remote regions beyond the exobase, since the Larmor radii of magnetospheric $\mathrm{O}^{+}$ions and heavy pick-up ions $\left(\mathrm{CH}_{4}^{+}\right.$and $\mathrm{N}_{2}^{+}$for example) are larger than the radius of the satellite itself. The importance of kinetic effects was first investigated with test particle simulations (Luhmann 1996; Ledvina et al. 2005). Newly born pick-up ions are accelerated by the motional electric field $\mathbf{E}_{\text {conv }}=-\mathbf{v} \times \mathbf{B}$ and move in cycloidal trajectories in a plane perpendicular to the undisturbed magnetospheric magnetic field (Hartle et al. 1982). The initial distribution of pick-up ions in velocity space is a ring distribution. This is the main reason why multi-fluid cannot take into account self-consistently the dynamic of pick-up, since these models assume a Maxwellian distribution function for ion species. However hybrid models do not make this assumption and can simulate the behavior of pick-up ions.

In a hybrid simulation, two different regions can be identified in Titan's wake, the main part of the planetary plasma wake which have an elliptical-like shape elongated in the direction of the undisturbed magnetic field and a second region more limited in size, associated to a plume-like structure with a density of fraction of ions per $\mathrm{cm}^{3}$. The main elliptical shape of Titan's plasma wake agrees well with MHD simulation (Backes et al. 2005). The plume of the so-called pick-up ions, occurs in a region governed by the magnetospheric plasma and is relatively narrow and aligned the motional electric field. In addition, the energy of these ions is significantly high (few keV). Outflowing ions in the main plasma wake region are cold (up to hundreds of eV). The difference in energy between the 'pick-up' region and the main plasma wake is due to the difference of the speed flow in these two regions. Ions in the main wake region are not accelerated as quickly as the pick-up ions in the magnetospheric region. This feature agrees well with the CAPS Cassini observations (Harnett and Winglee 2006).

Moreover, the multi-species observed by Cassini observations are reproduced well by hybrid models. While multi-fluid models have to resolve a set of equation for each ion species in order to take into account the difference in density, speed and temperature, hybrid models include multiple species by adjusting the mass ratio for the different macroparticles. The main limitation of hybrid models is that a large number of particles must be included to obtain reasonable statistical sampling of each ions species.

Figure 4 emphasizes the different dynamic behavior of each ion species. Density maps of magnetospheric plasma (Fig. 4(a)-(c)) show that $\mathrm{O}^{+}(2 \mathrm{keV})$ and energetic $\mathrm{H}^{+}(30 \mathrm{keV})$ do not penetrate below the exobase level, except in a limited region where energetic magnetospheric ions can precipitate (Fig. 4(a), (c)). However, hybrid models are usually not well designed to study collisional region such as the atmosphere (Modolo and Chanteur 2008). On the other hand thermal protons $(200 \mathrm{eV})$ show a completely different pattern and are mainly excluded from the induced magnetospheric region (Fig. 4(b)), similarly to the Martian picture. Energetic protons have larger gyroradii than thermal protons and thus are less 
E. Kallio et al.
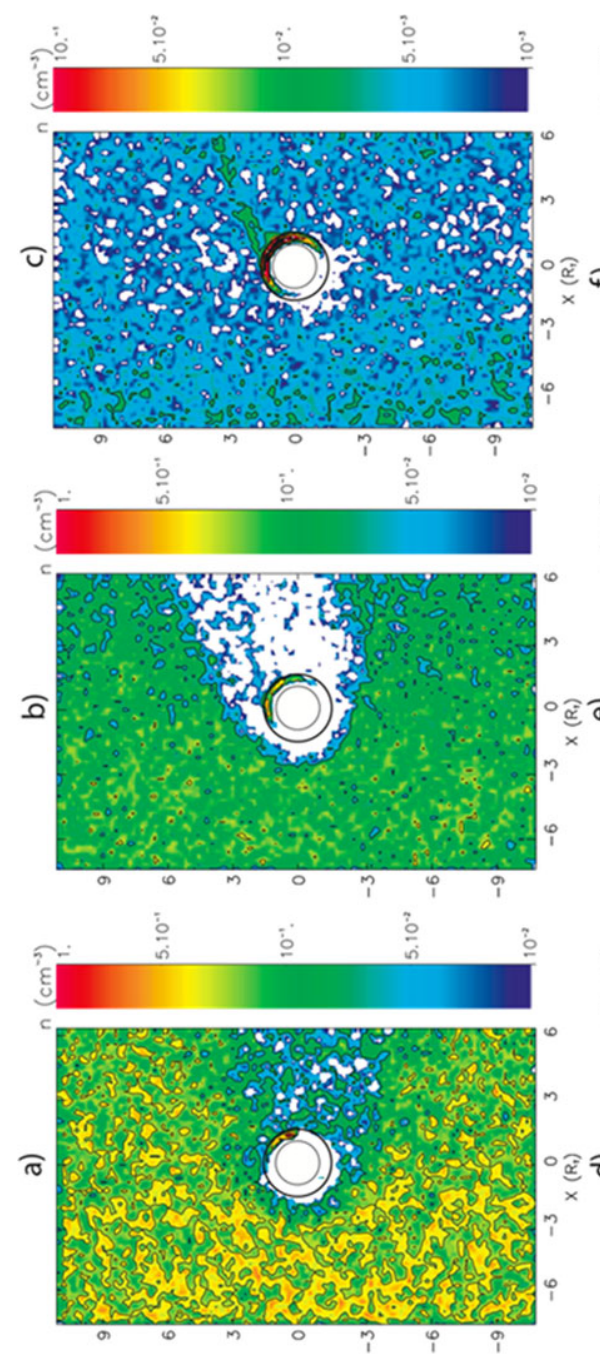

('y) $\lambda$
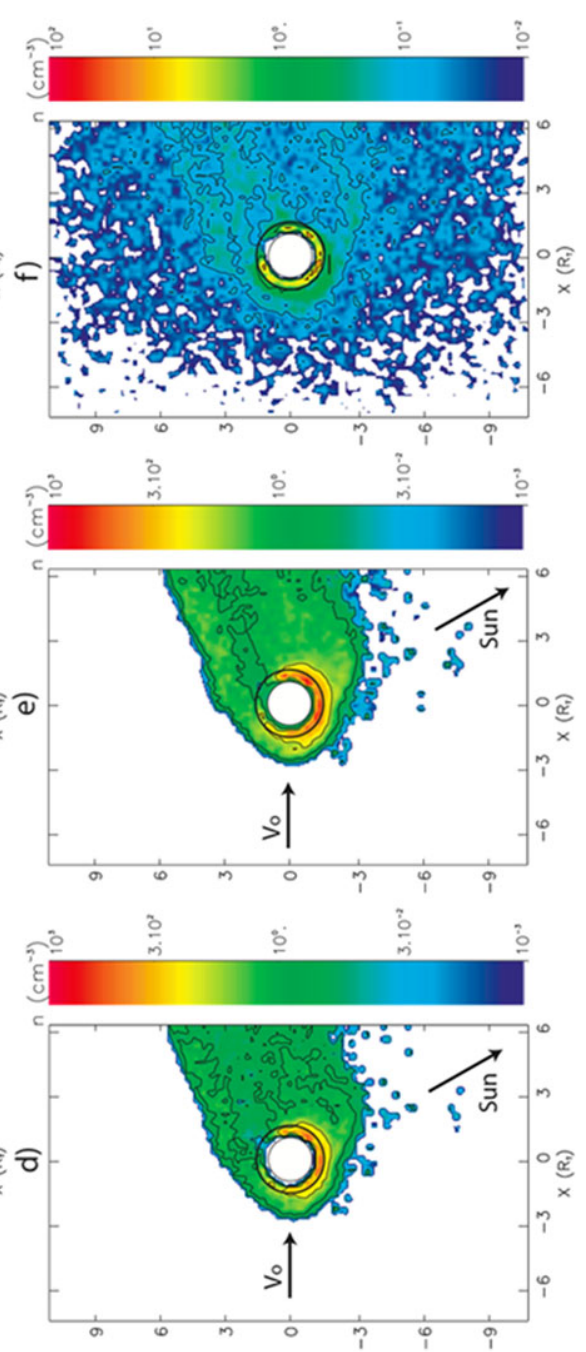

('d) 1

$\stackrel{g}{g}$

产声

急

क

혀웅

을

施

힠

肎

흥흐

ᄋ묘

纪

-.

․․․․․

40

늘

동

를

ㅡㄹ

=

읭 형

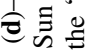

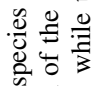

동

赔左

要.

吅密

过

क्षे 웜

ชำ

害令

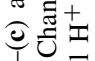

ङ

\& 응

ปั

की

.을

늘

त्ञ

늘

해용

可

뚜

음

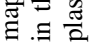

궁.

竞

อั

$\checkmark$ 造证

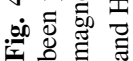


sensitive to draped magnetic field lines, since they required more time to adjust their trajectory to the magnetic field direction. The planetary plasma is mainly confined in the induced magnetospheric region (Figs. 4(d)-(f)). The largest ionospheric density is observed on the sunlight side of the ionosphere. The heavy planetary plasma $\left(\mathrm{CH}_{4}^{+}\right.$and $\left.\mathrm{N}_{2}^{+}\right)$extends $2.5-3$ Titan radii on the ram-side and fills the plasma wake of the induced magnetospheric region while the planetary light ion $\left(\mathrm{H}_{2}^{+}\right)$shows a larger spread even in the magnetospheric region, and contributes to the mass loading region (Fig. 4(f)). The dissimilarity between light and heavy ion structure can be explained by the difference of the production source. The main source of ionization for $\mathrm{H}_{2}^{+}$is charge exchange, efficient in the magnetospheric region, while the main source of ionization for heavy planetary ions is photoionization and electron impact ionization (Modolo et al. 2007).

\subsubsection{Hybrid Models of Venus' Interaction with the Solar Wind}

Only a few hybrid models have been adapted to study Venus' plasma interaction. One of the main reasons is that the size of the obstacle is much larger than the typical solar wind ion scale. Therefore, the interaction is more fluid-like.

Moore et al. (1991) developed the first two-dimensional hybrid model used to study the solar wind interaction with Venus. It emphasized the asymmetry of the $\mathrm{O}^{+}$dynamics in the direction of the motional electric field, which is in many ways similar to the Martian case. With a three-dimensional model, Kallio et al. (2006) showed that asymmetry in the escape of $\mathrm{O}^{+}$was due to the motional electric field, in agreement with Moore et al. (1991). Hybrid simulations are in good agreement with Pioneer Venus Orbiter observations (Jarvinen et al. 2008) and Venus-Express observations (Kallio et al. 2008; Zhang et al. 2010) and reproduce the main boundaries and regions.

\subsection{Fully Kinetic Methods}

Fully kinetic models provide a comprehensive description of the plasma. This method has not yet been used in global VMT model but it is informative to describe its basic properties for a theoretical completeness.

In fully kinetic model, all ions and electrons are modeled as particles that are accelerated by the Lorentz force and possible non-Lorentz forces $(\mathbf{F})$ :

$$
\begin{aligned}
& \frac{d \mathbf{v}_{p}}{d t}=\frac{q_{p}}{m_{p}}\left(\mathbf{E}+\mathbf{v}_{p} \times \mathbf{B}\right)+\mathbf{F} \\
& \frac{d \mathbf{x}_{p}}{d t}=\mathbf{v}_{p}
\end{aligned}
$$

Here $\mathbf{v}_{p}, \mathbf{x}_{p}, m_{p}$ and $q_{p}$ are the velocity, position, mass and charge of an ion or electron denoted by the subscript $p$. The forces in (2.33) can include gravitational forces and forces associated with ion-ion, ion-electron, ion-neutral or electron-neutral collisions.

The magnetic field is propagated in time by Faraday's law

$$
\frac{\partial \mathbf{B}}{\partial t}=-\nabla \times \mathbf{E}
$$


Fig. 5 A three-dimensional spherical electromagnetic wave in a fully kinetic model. The run includes an oscillating point source in a vacuum located at the center of the figure and the source generates a sinusoidal electric field. The white lines and the yellow lines are the electric and the magnetic field lines, respectively, which are added to illustrate the electromagnetic field in 3D. The vantage point is slightly below of the source in order to see the circular magnetic field-lines about the vertical axis. (Pohjola and Kallio 2010)

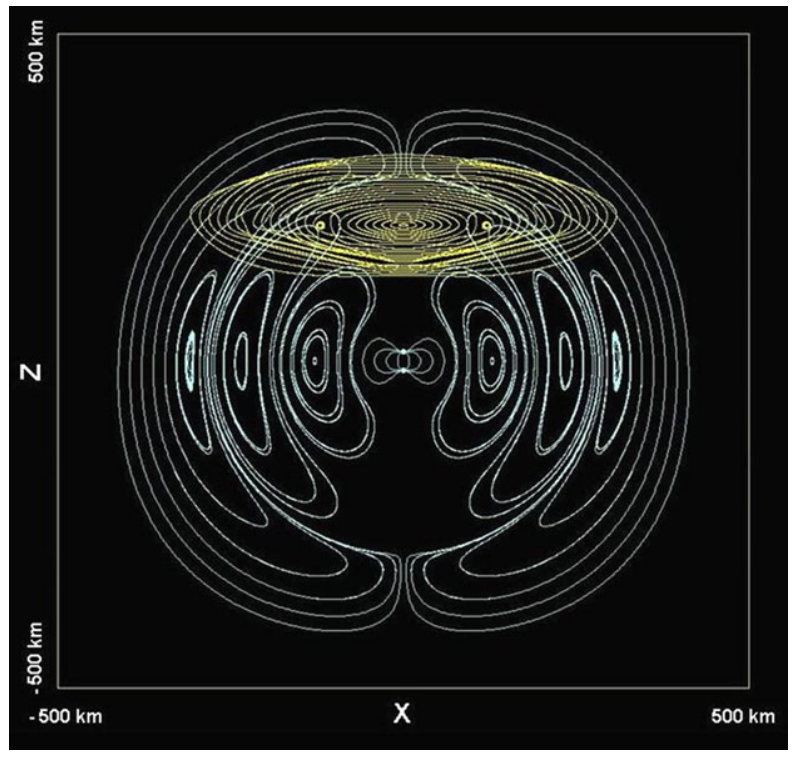

and the electric field by Ampere-Maxwell's law

$$
\frac{\partial \mathbf{E}}{\partial t}=\nabla \times \mathbf{B} / \varepsilon_{o} \mu_{o}-\mathbf{j} / \varepsilon_{o} .
$$

In (2.36), $\mathbf{j}, \varepsilon_{o}$ and $\mu_{o}$ are the electric current, the electric permittivity and the magnetic permeability, respectively.

As seen in from (2.35) and (2.36), the plasma is coupled to $\mathbf{E}$ and $\mathbf{B}$ fields through the electric current. There are several ways to derive $\mathbf{j}$ from particles. The electric current can be derived from the macroscopic plasma parameters:

$$
\mathbf{j}=\sum_{i} q_{i} n_{i} \mathbf{V}_{i}-e n_{e} \mathbf{V}_{e}
$$

where the subscript $i$ refers to ions and $e$ to electrons, $\mathbf{V}$ and $n$ are the bulk velocity and the number density, $q_{i}$ is the charge of a ion and $e$ is the elementary charge $\left(1.6 \times 10^{-19} \mathrm{C}\right)$ (see, e.g. Pohjola and Kallio 2010). The plasma densities and bulk velocities can be derived from the individual particle positions and velocities (Pohjola and Kallio 2010) but also other techniques can be used (see Umeda 2010, and ref. therein).

As can be seen from (2.33)-(2.37), the basic equations are straightforward, however several issues make solving these equations challenging. First, (2.36) can be rearranges to include the displacement current term $\left(\equiv \varepsilon_{o} \mu_{o} \frac{\partial \mathbf{E}}{\partial t}\right)$. Therefore, fully kinetic models include high frequency electromagnetic waves which can lead to numerical instabilities. Figure 5 shows an example of a fully kinetic simulation in which a point source is emitting electromagnetic waves in a vacuum.

Secondly, fully kinetic models do not require the assumption of quasi-neutrality, that is, the assumption that the electron density is equal the total charge density of ions. This means that the electric field can be derived from Ampere-Maxwell's law (Eq. (2.36)) and not from the electron momentum equation as in the hybrid model (cf. Sect. 2.2). It is also important to note that if the electric field is derived from (2.36), then Gauss' law does not 


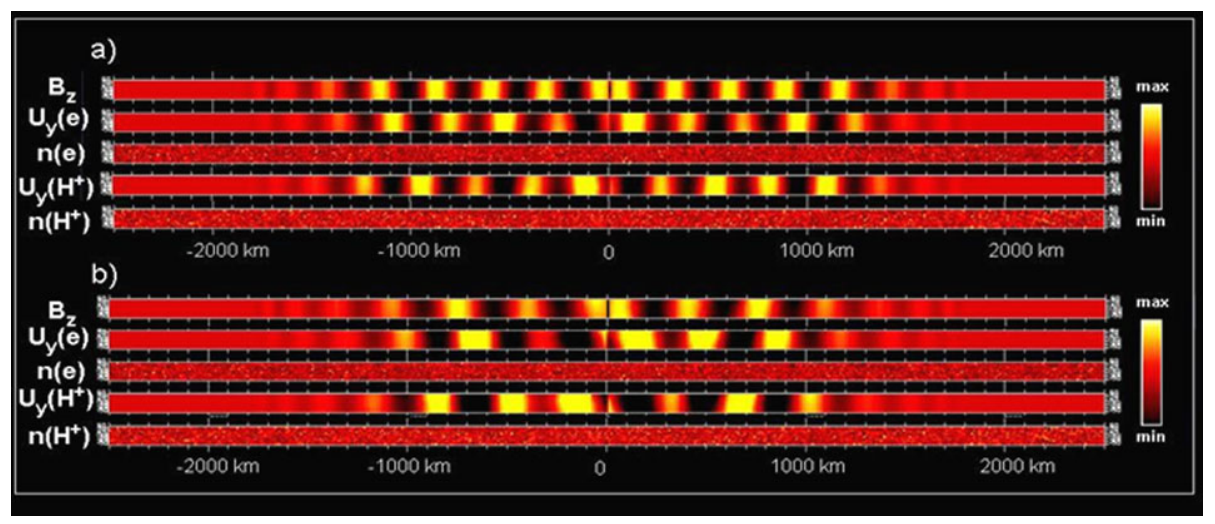

Fig. 6 The propagation of an electromagnetic wave in a electron-proton plasma based on a fully kinetic model. The horizontal axis is the $x$-axis and a plane wave is generated at $x=0$. In panel (a) the initial electron density in the simulation box was $0.016 \mathrm{~cm}^{-3}$ and in panel (b) it was $0.021 \mathrm{~cm}^{-3}$. In panel (a) the tables from top to bottom are: $B_{z}$ from $-1 \times 10^{-12} \mathrm{~T}$ to $1 \times 10^{-12} \mathrm{~T}$, the velocity of electrons in the $y$-direction $\left(U_{y}(e)\right)$ from $-10 \mathrm{~km} / \mathrm{s}$ to $10 \mathrm{~km} / \mathrm{s}$, the electron density $(n(e))$ from 0 to $0.03 \mathrm{~cm}^{-3}$, the velocity of protons in the $y$-direction $\left(U_{y}\left(\mathrm{H}^{+}\right)\right)$from $-5 \mathrm{~m} / \mathrm{s}$ to $5 \mathrm{~m} / \mathrm{s}$ and the proton density $\left(n\left(\mathrm{H}^{+}\right)\right)$from 0 to $0.03 \mathrm{~cm}^{-3}$. In panel (b) the tables from top to bottom are: $B_{z}$ from $-1 \times 10^{-12} \mathrm{~T}$ to $1 \times 10^{-12} \mathrm{~T}, U_{y}(e)$ from $-10 \mathrm{~km} / \mathrm{s}$ to $10 \mathrm{~km} / \mathrm{s}, n(e)$ from 0 to $0.04 \mathrm{~cm}^{-3}, U_{y}\left(\mathrm{H}^{+}\right)$from $-5 \mathrm{~m} / \mathrm{s}$ to $5 \mathrm{~m} / \mathrm{s}$ and $n\left(\mathrm{H}^{+}\right)$from 0 to $0.04 \mathrm{~cm}^{-3}$. In both panels the color map on the right hand side is a linear color map from the minimum value (min) of the variable to the maximum value (max) of the variable (Pohjola and Kallio 2010)

need to be solved explicitly. It can be seen, by taking the divergence of Ampere-Maxwell's law (Eq. (2.36)), that Gauss' law is fulfilled during the simulation (if it is fulfilled initially). However, deriving the electric field this way means that the model must solve the continuity equation (e.g. Pohjola and Kallio 2010), which can be done by adopting a specific accurate charge accumulation scheme (e.g. Villasenor and Buneman 1992, or Umeda et al. 2003) or by cleaning the electric field with a Poisson solver (Birdsall and Langdon 1985).

Third, the spatial (ion inertial length and gyroradius) and temporal (gyrofrequency and plasma frequency) scales of a fully kinetic model are determined by both the mass of ions and electrons. Electron spatial and temporal scales are very small compared to the ion scales because of the mass difference between the electron and ions. To properly simulate electron inertial scales and the electron plasma and gyro frequency, one has to use a small grid size and small time step. One also has to adopt small spatial and temporal scales to model fast propagating electromagnetic waves and to reach the Debye length scale. In plasma physics one way to speed up the simulations in a specific application is to replace the fully electromagnetic code described above by a so-called Darwin electromagnetic code, where the transverse displacement current is omitted from (2.36), or to use implicit time integration (see, for example, Langdon 1986; Birdsall and Langdon 1985; Besse et al. 2007). These limitations mean that, in practice, fully kinetic models are more applicable for small-scale local simulations rather than large-scale global simulations.

However, fully kinetic models contain very rich physics and do not contain any "subscales". Fully kinetic models also include fewer approximations than hybrid and MHD models and give a more comprehensive description of particles dynamics and electromagnetic fields. For example, fully kinetic models can be used to study the stability of various planetary plasma regions in three-dimensional space. They can also be used to analyze the propagation of waves in various plasma environments. Figure 6 shows results from a fully kinetic 
simulation of electromagnetic waves propagating in plasma. Similarly, fully kinetic models could be used to study the propagation of naturally or artificially produced electromagnetic waves in planetary ionospheres or surfaces.

\section{Models of Mars' Neutral Exosphere}

Developing a comprehensive global model of the interaction of the atmosphere of VMT and their space environments is challenging because the model should include not only charged particles but also neutrals particles. The flowing plasma near VMT interacts directly with the atmospheres of the non-magnetized bodies; therefore, a comprehensive model has to take into account charged particle-neutral reactions. Moreover, ions are generated from the neutrals by charge exchange processes, electron impact ionization, photoionization and chemical processes. A comprehensive unified model should therefore contain both a global plasma model and a global model for planetary neutrals. The goal of this section is to describe how this approach has been used to model the exosphere of Mars.

\subsection{The Neutral Exosphere of Mars}

The exosphere is the collisionless region of the upper atmosphere, extending from the exobase (near $200 \mathrm{~km}$ altitude for Mars) into interplanetary space. The exosphere of Mars is important by itself but it is also important because it is formed through the same processes that cause atmospheric escape. Therefore, understanding Mars' exosphere helps us understand Martian atmospheric escape and its effect on Martian climatic change (Chassefière et al. 2007). This section can be considered as a complement of the recent review about escape modeling done by Johnson et al. (2008), which focuses on the Martian neutral exosphere.

The exosphere of Mars is composed of two oxygen populations, a cold or thermal population produced by vertical diffusion of oxygen atoms from the lower thermosphere and a hot population resulting from the dissociative recombination of the $\mathrm{O}_{2}^{+}$ions in the upper thermosphere and exosphere (McElroy 1972; Nagy and Cravens 1988). The hot oxygen atoms formed by dissociative recombination of $\mathrm{O}_{2}^{+}$can produce a recoil population through a cascade of collisions with the thermal oxygen population (Shematovich et al. 1994). Other sources of hot neutral oxygen such as sputtering, due to the precipitation of accelerated pick-up $\mathrm{O}^{+}$ions into the Martian atmosphere, are less important in current conditions (Chaufray et al. 2007) but could have played a more important role in the past (Leblanc and Johnson 2001; Johnson et al. 2008). Moreover, recent detections of energetic neutral atoms (ENAs) produced by charge exchange between energetic ions and the neutral exosphere require accurate coupling between the neutral exosphere and solar wind (Futaana et al. 2011). It is therefore important to couple an exospheric model to a plasma model to accurately describe processes related to the solar wind interaction but also to study the effect of pick-up ions precipitation into the Martian upper atmosphere and the additional escape produced by sputtering. This may be particular important when studying past escape on Mars.

The $\mathrm{O}_{2}^{+}$dissociative recombination is divided into 5 channels:

$$
\begin{array}{r}
\mathrm{O}_{2}^{+} \rightarrow \mathrm{O}\left({ }^{3} \mathrm{P}\right)+\mathrm{O}\left({ }^{3} \mathrm{P}\right)+\Delta E=6.95 \mathrm{eV}(0.22 \pm 0.10) \\
\mathrm{O}\left({ }^{1} \mathrm{D}\right)+\mathrm{O}\left({ }^{3} \mathrm{P}\right)+\Delta E=4.99 \mathrm{eV}(0.42 \pm 0.05)
\end{array}
$$


Fig. 7 Production rates of the $\mathrm{O}_{2}^{+}$ground state dissociative recombination function of the temperature from Mehr and Biondi (1969) (solid line) and Peverall et al. (2001) (dashed line)

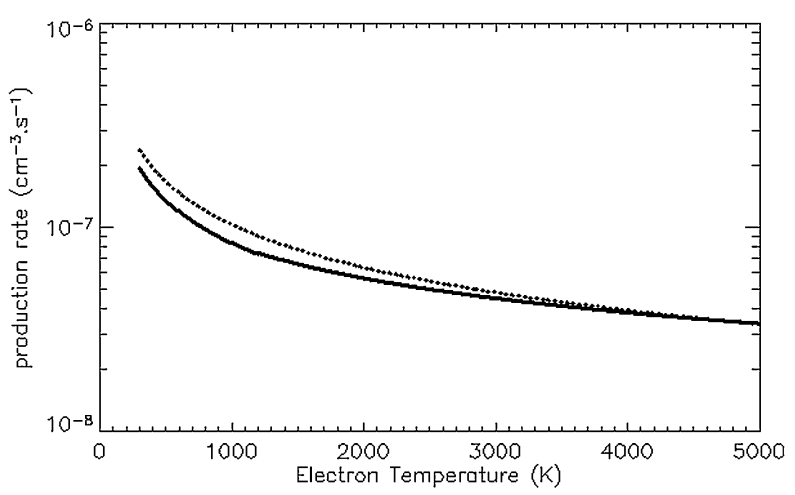

$$
\begin{aligned}
& \mathrm{O}\left({ }^{1} \mathrm{D}\right)+\mathrm{O}\left({ }^{1} \mathrm{D}\right)+\Delta E=3.02 \mathrm{eV}(0.31 \pm 0.07) \\
& \mathrm{O}\left({ }^{3} \mathrm{P}\right)+\mathrm{O}\left({ }^{1} \mathrm{~S}\right)+\Delta E=2.77 \mathrm{eV}(<0.01) \\
& \mathrm{O}\left({ }^{1} \mathrm{D}\right)+\mathrm{O}\left({ }^{1} \mathrm{~S}\right)+\Delta E=0.80 \mathrm{eV}(0.05 \pm 0.02)
\end{aligned}
$$

For the ground state, the branching ratios have been measured by Kella et al. (1997) using the ASTRID heavy ion storage ring and are given in parenthesis. The fourth branching ratio has efficiency equal to 0 (Kella et al. 1997). The energy in the center of mass is indicated for each channel (Kella et al. 1997). If this energy is divided equally between the two oxygen atoms produced, only the first two channels will produce atoms with energy larger than the escape velocity $(\sim 2 \mathrm{eV}$ at the exobase). The production rates, the branching ratios and the exothermicity, $\Delta E$, of the reaction depend on the vibrational state of the $\mathrm{O}_{2}^{+}$ions (Guberman 1983, 1989). The addition of one vibrational quantum adds about $0.23 \mathrm{eV}$ to the exothermicity (Fox and Hac 1997). The vibrationally excited states of $\mathrm{O}_{2}^{+}$ions are controlled by ion-neutral reactions in the thermosphere (Fox and Hac 1997) and their altitude distributions have been modeled in the Martian ionosphere by Fox and Hac (2009) at a $60^{\circ}$ solar zenith angle (see also Futaana et al. 2011, this issue). The production rates from Mehr and Biondi (1969) and Peverall et al. (2001) are displayed in Fig. 7 for typical electron temperatures in the Martian upper atmosphere between 300 and $5000 \mathrm{~K}$. There is a systematic difference of $20 \%$ below $1200 \mathrm{~K}$ and a good agreement for electron temperature larger than $3000 \mathrm{~K}$.

Mars' cold oxygen population has been observed through its $130 \mathrm{~nm}$ emission line (Barth et al. 1971; Strickland et al. 1972, 1973; Stewart et al. 1992; Leblanc et al. 2006; Chaufray et al. 2009) at altitudes below $400 \mathrm{~km}$. The profile of the $130 \mathrm{~nm}$ oxygen emission observed by ALICE onboard Rosetta during its Mars flyby present a change in slope, indicating two oxygen populations (Feldman et al. 2011). The cold oxygen population is dominant below $500 \mathrm{~km}$ while the hot oxygen population is dominant above $500 \mathrm{~km}$ (Feldman et al. 2011). In the last decades, several models have been developed to study the Martian exosphere. Most of the work has focused on oxygen density. No model currently describes, in a single consistent approach, the full exospheric oxygen distribution. Currently, exospheric models solve Boltzmann equations for cold and hot oxygen populations separately. Several approaches have been used to study the hot oxygen population such as the two-stream model method (Nagy and Cravens 1988) or Monte Carlo models (Hodges et al. 2000). In the 1D two-stream approach, the hot oxygen flux is divided into an upward flux $\Phi^{+}(E, z)$ and a downward flux $\Phi^{-}(E, z)$ where $z$ is the altitude and $E$ the energy of the oxygen atoms. The linear coupled equations of these two fluxes are solved below the exobase and the velocity distribution 
function at the exobase altitude $z_{c}$ is computed as $f(V)=\left[\Phi^{+}\left(E, z_{c}\right)+\Phi^{-}\left(E, z_{c}\right)\right] / V(E)$ where $V$ is the velocity associated to the energy $E$. Then, the Liouville equation is solved above the exobase to describe the density in the exosphere (Zhang et al. 1993; Kim et al. 1998). In Monte Carlo models, the hot oxygen atoms simulated by "representative" particles or test particles, which are followed along their trajectory. This approach uses more realistic 2D or 3D thermospheric-ionospheric environments (Hodges 2000; Leblanc and Johnson 2002; Chaufray et al. 2007; Valeille et al. 2009a, 2009b) or realistic cross sections to describe the collision between hot oxygen atoms and the background atmosphere (Krestyanikova and Shematovich 2005; Cipriani et al. 2007; Fox and Hac 2009). The collision cross sections of hot oxygen and cold thermospheric species are crucial parameters, as shown by several studies (Shizgal 1999a; Johnson et al. 2000; Cipriani et al. 2007; Fox and Hac 2009; Lichtenegger et al. 2009).

While numerous studies have analyzed hydrogen in Earth's (e.g., Chamberlain 1963, 1977, 1980; Vidal-Madjar and Bertaux 1972; Bertaux 1978; Shizgal and Blackmore 1986; Hodges 1994) and Venus' exosphere (e.g., Rodriguez et al. 1984; Shizgal 1987; Nagy et al. 1990; Hodges 1999; Shizgal 1999a), few studies have described Mars' hydrogen exosphere. Nagy et al. (1990) studied the production of hot hydrogen produced by charge exchange using a two-stream model approach. Shizgal (1999a) studied the hydrogen and deuterium escape caused by energization due to collisions with hot oxygen but focused more on Venus' exosphere rather than Mars' exosphere due to the lack of observations. Chen and Cloutier (2003) developed a 3D Monte Carlo model that included radiation pressure, photoionization and charge exchange with solar wind protons and found density profiles similar to Chamberlain's approach without satellite particles. More recently Lichtenegger et al. (2006) used a Monte Carlo model to estimate the energy distribution of hot hydrogen at the exobase. They considered the exothermic reactions in McElroy et al. (1982) and found that hot hydrogen was negligible on Mars.

A comparative study of the Venusian and Martian hydrogen exospheres is needed since the differences and similarities have not been examined since the work of Nagy et al. (1990) who did not consider the full set of reactions. First, we will present Chamberlain's method, which is usually used to describe the hydrogen in the Martian exosphere, and then the Monte Carlo approach, which widely used to study the hot oxygen population in the Martian exosphere.

\subsection{Chamberlain's Method}

Chamberlain's approach (Chamberlain 1963) is based on the solution of the Liouville equation in a collisionless exosphere. Once this equation is solved, the velocity distribution is integrated in the region of velocity space consistent with trajectories coming from the exobase in a gravitational field. The velocity space can be divided into different regions, which represent different classes of trajectories (Fahr and Shizgal 1983). Figure 8 displays a cut of the velocity space. The conditions for each class of trajectory are summarized in Table 2 . In this section, a particle will be identified by its trajectory. For example, an escaping particle is a particle with an escaping trajectory.

The parameters (using the notation of Chamberlain 1963) are defined as follows, $\lambda$ is the escape parameter defined by

$$
\lambda(r)=\frac{G M m}{k T_{c} r}=\frac{V_{\mathrm{esc}}^{2}}{U^{2}},
$$


Fig. 8 Repartition of the different classes of particle in the velocity space adapted from Fahr and Shizgal (1983). The reduced velocity $w$ is defined as the ratio of the velocity and the thermal velocity. The parameters $\lambda, \psi_{1}$ and $\theta$ are defined by (3.6), (3.7), (3.8) respectively

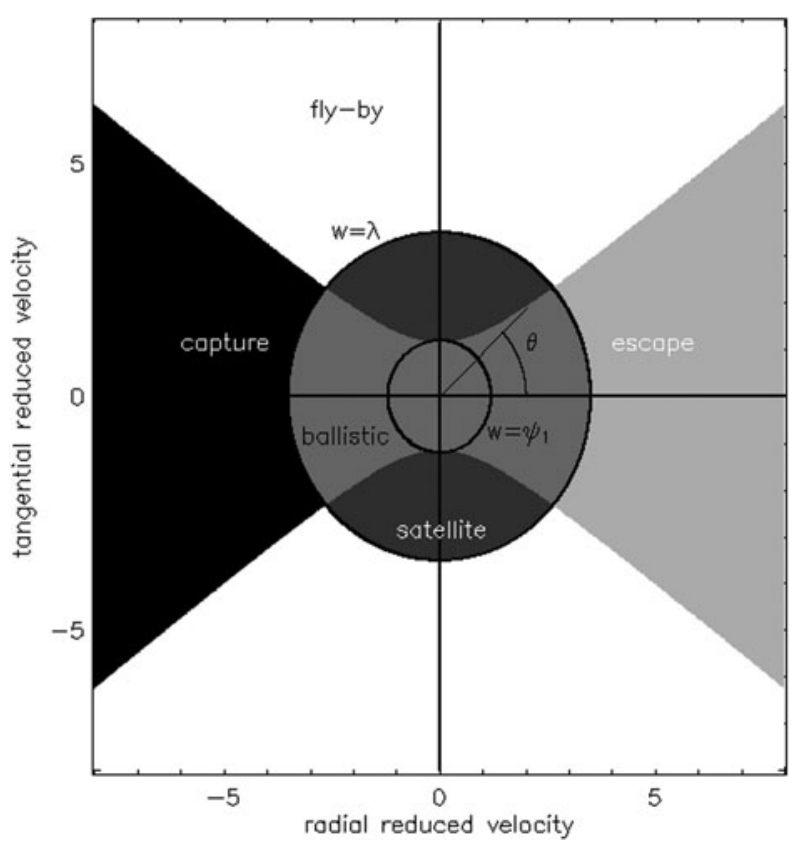

Table 2 Different classes of particles and their distribution in the velocity space, adapted from Fahr and Shizgal (1983)

\begin{tabular}{|c|c|c|c|c|}
\hline $\begin{array}{l}\text { Class of } \\
\text { particle }\end{array}$ & Orbit & $w_{\text {range }}$ & $\theta_{\text {range }}$ & Domain of velocity space \\
\hline Balistic (B) & $\begin{array}{l}\text { Elliptic } \\
\text { crossing the } \\
\text { exobase }\end{array}$ & $\begin{array}{l}0 \leq w \leq \psi_{1}^{1 / 2} \\
\psi_{1}^{1 / 2} \leq w \leq \lambda^{1 / 2}\end{array}$ & $\begin{array}{l}0 \leq \theta \leq \pi \\
0 \leq \theta \leq \theta_{m}(w)\end{array}$ & $\begin{array}{l}\text { Between the two circles and } \\
\text { outside the hyperbolae }\end{array}$ \\
\hline Satellite (S) & $\begin{array}{l}\text { Elliptic not } \\
\text { crossing the } \\
\text { exobase }\end{array}$ & $\psi_{1}^{1 / 2} \leq w \leq \lambda^{1 / 2}$ & $\theta_{m}(w) \leq \theta \leq \pi$ & $\begin{array}{l}\text { Inside the largest circle and } \\
\text { inside the hyperbolae }\end{array}$ \\
\hline$B+S$ & Elliptic & $0 \leq w \leq \lambda^{1 / 2}$ & $0 \leq \theta \leq \pi$ & Inside the largest circle \\
\hline Escaping $(\mathrm{E})$ & $\begin{array}{l}\text { Hyperbolic } \\
\text { crossing the } \\
\text { exobase }\end{array}$ & $\begin{array}{l}w>\lambda^{1 / 2} \\
w_{r}>0\end{array}$ & $0 \leq \theta \leq \theta_{m}(w)$ & $\begin{array}{l}\text { Outside the largest circle } \\
\text { and outside the hyperbolae } \\
\text { in the field } w_{r}>0\end{array}$ \\
\hline Capture (C) & $\begin{array}{l}\text { Hyperbolic } \\
\text { crossing the } \\
\text { exobase }\end{array}$ & $\begin{array}{l}w>\lambda^{1 / 2} \\
w_{r}<0\end{array}$ & $0 \leq \theta \leq \theta_{m}(w)$ & $\begin{array}{l}\text { Outside the largest circle } \\
\text { and the hyperbolae, in the } \\
\text { field } w_{r}<0\end{array}$ \\
\hline Fly-by & $\begin{array}{l}\text { Hyperbolic } \\
\text { not crossing } \\
\text { the exobase }\end{array}$ & $w>\lambda^{1 / 2}$ & $\theta_{m}(w) \leq \theta \leq \pi$ & $\begin{array}{l}\text { Outside the largest circle } \\
\text { and inside the hyperbolae }\end{array}$ \\
\hline $\mathrm{E}+\mathrm{C}+\mathrm{F}$ & Hyperbolic & $w>\lambda^{1 / 2}$ & $0 \leq \theta \leq \pi$ & Outside the largest circle \\
\hline
\end{tabular}

where $G$ is the gravitational constant, $M$ the mass of the planet, $m$ the mass of the specie, $k$ the Boltzmann constant, $T_{c}$ the exobase temperature, $r$ the radial distance from the center of the planet, $V_{\text {esc }}$ the escape velocity and $U=\left(2 k T_{c} / m\right)^{1 / 2}$ is the thermal velocity. The 
equation of the hyperbolae delimitating the satellite and flyby particles from the escape, ballistic and capture particles is

$$
w_{t}^{2}-\frac{\lambda^{2}}{\lambda_{c}^{2}-\lambda^{2}} w_{r}^{2}=\frac{\lambda^{2}}{\lambda+\lambda_{c}}=\psi_{1},
$$

where, $w_{r}$ and $w_{t}$ are the radial and the tangential components of the reduced velocity which is the ratio between the velocity and the thermal velocity $U$. The parameter $\theta_{m}$ is given by

$$
\cos \theta_{m}(w)=\frac{\left(\lambda_{c}^{2}-\lambda^{2}\right)^{1 / 2}}{\lambda_{c}}\left(1-\frac{\psi_{1}}{w^{2}}\right)^{1 / 2}=\left[1-\frac{\lambda^{2}}{\lambda_{c}^{2}}\left(1+\frac{\lambda_{c}-\lambda}{w^{2}}\right)\right]^{1 / 2} .
$$

In the exosphere, if collisions were totally neglected, only particles with trajectories crossing the exobase (ballistic and escaping) would be present. To take into account long-lived satellite particles, which can exist in the lower exosphere due to rare collisions, Chamberlain (1963) introduced the satellite critical radius. Satellite particles are included below this radius and excluded above it.

In spherically symmetric exospheres without collisions, the Liouville equation can be written under the form

$$
v_{r} \frac{\partial f}{\partial r}+\left(\frac{P_{x}^{2}}{m r^{3}}-\frac{G M m}{r^{2}}\right) \frac{\partial f}{\partial p_{r}}=0,
$$

where $(r, \theta)$ are the standard spherical coordinates, $P_{\chi}=m r^{2}(d \theta / d t)$ is a constant according to Kepler's second law, $f$ is the velocity distribution function, $v_{r}$ is the radial velocity and $p_{r}=m v_{r}$. Assuming a function with a form $f=A(r) \exp \left(-p_{r}^{2} / 2 m k T_{c}\right)$, the equation can be written as

$$
\frac{d(\ln f)}{d r}=-\left(\frac{G M m}{k T_{c} r^{2}}-\frac{P_{x}^{2}}{M k T_{c} r^{3}}\right) .
$$

This equation is then solved using a Maxwellian distribution as the boundary condition at the exobase. The velocity distribution at an altitude $r$ is therefore given by

$$
f\left(r, p_{r}, P_{x}\right)=\frac{n_{c} \exp \left(-\left(\lambda_{c}-\lambda\right)\right) \exp \left(-p_{r}^{2} / 2 m k T_{c}\right) \exp \left(-P_{x}^{2} / 2 m k T_{c} r^{2}\right)}{\left(2 \pi k T_{c} / m\right)^{3 / 2}},
$$

The velocity distribution is integrated over the velocity region allowed by the trajectory in a gravitational field (Table 2) and leads to the density given by

$$
n(r)=n\left(r_{c}\right) \exp \left[-\left(\lambda_{c}-\lambda\right)\right] \zeta(\lambda)
$$

where $\zeta(\lambda)$ equal to 1 when the distribution given by (3.11) is integrated over the full velocity space leading to the hydrostatic density profile. This means that the barometric law, usually derived from equilibrium between gas pressure and gravitation (and should be valid only below the exobase) is still valid in the exosphere provided the moments extend over all possible values and assuming a Maxwellian distribution at the exobase (Chamberlain 1963).

Analytical expressions of the $\zeta$ function for each class of particle can be found in Chamberlain (1963). The Jeans escape formula can be derived from the integration of the first moment of the velocity function distribution over the escape particles velocity space do- 


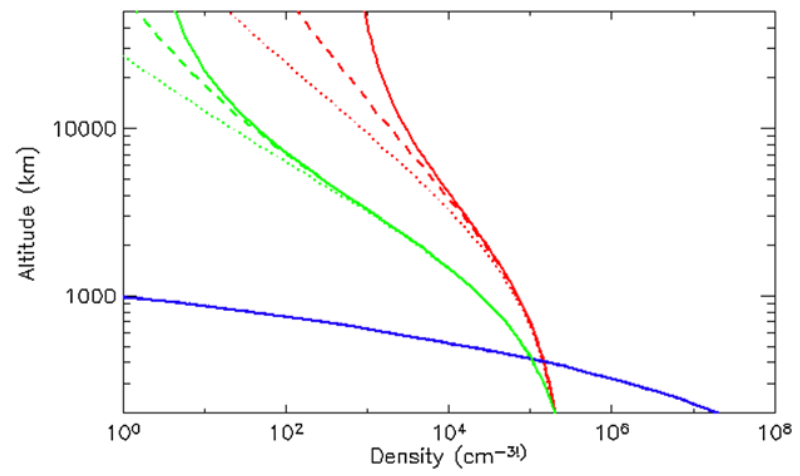

Fig. 9 Examples of density profiles for atomic hydrogen (red), molecular hydrogen (green) and atomic oxygen (blue) derived under different assumptions for an exobase temperature of $250 \mathrm{~K}$. Solid lines represent the barometric law with the specie scale height, dashed lines represent density profiles including ballistic, satellite and escaping particles, dotted lines represent the density profile including only ballistic and escaping particles

main (Chamberlain 1963). Examples of hydrogen density profiles under different assumptions are displayed in Fig. 9 with $T_{c}=250 \mathrm{~K}$. For atomic oxygen there is no difference in the density profile when all classes of particles are included (hydrostatic) and when only escape and ballistic particles are included. For atomic hydrogen the hydrostatic assumption overestimates the density above $2000 \mathrm{~km}$ compared to the density derived with only escape and ballistic particles, while including satellite particles changes the distribution above $4000 \mathrm{~km}$. For $\mathrm{H}_{2}$, departure from the hydrostatic law occurs near $4000 \mathrm{~km}$ altitude. The hydrostatic assumption used by some 1D collisional models above the exobase, until $400 \mathrm{~km}$ (e.g. Krasnopolsky 2010), is therefore justified. Above $\sim 400$ to $500 \mathrm{~km}$, nonthermal populations, especially non-thermal oxygen, cannot be neglected (Valeille et al. 2009a).

\subsection{Monte Carlo Method}

The first Monte Carlo simulations of planetary exospheres were performed to determine how to correct Jeans' escape so that it takes into account the perturbation of the velocity distribution due to escaping $\mathrm{H}$ and $\mathrm{He}$ at the exobase of Earth (see Brinkman 1971 and references therein). Later, this approach was used to model the structure of the hydrogen and helium exospheres of the Moon and Mercury (Hodges 1973, 1980; Curtis and Hartle 1978; Smith et al. 1978). Since then, Monte Carlo models have been used extensively to study different processes in several exospheric environments. In this section, we illustrate the Monte Carlo approach, by describing the models used to study the oxygen exosphere and escape rate on Mars.

Monte Carlo simulations have been used to describe exospheric hot oxygen density and escape produced by the dissociative recombination of $\mathrm{O}_{2}^{+}$ions (Hodges 2000; Krestyanikova and Shematovich 2005; Cipriani et al. 2007; Chaufray et al. 2007; Valeille et al. 2009a; Fox and Hac 2009) and sputtering (Leblanc and Johnson 2001, 2002; Cipriani et al. 2007; Chaufray et al. 2007). The main inputs of Monte Carlo models are the thermosphericionospheric background and collisions cross-sections between hot oxygen atoms and thermospheric species (mainly $\mathrm{CO}_{2}$ and thermal $\mathrm{O}$ ). Table 3 summarizes the assumptions used by different authors and the processes described. 
Table 3 Assumptions made by the recent Monte Carlo studies of the hot oxygen coronae. DR: Dissociative Recombination of $\mathrm{O}_{2}^{+}$ions, CE: Charge Exchange between energetic $\mathrm{O}^{+}$ions and ambient $\mathrm{H}$ or O. HS: Hard Sphere, UP: Universal Potential, MD: Molecular Dynamics Model from Johnson et al. (2002), FSDCS: Forward Scattering Differential Cross Section. 1D means that density depends only on the radius $r, 2 \mathrm{D}$ means a dependence on $r$ and $s z a$ (solar zenith angle). For sputtering models, the ionosphere is not needed

\begin{tabular}{|c|c|c|c|}
\hline Authors & Processes & Thermosphere/Ionosphere & Collisions \\
\hline Hodges et al. (2000) & $\mathrm{DR}, \mathrm{CE}$ & 2D/2D, winds, rotation & HS \\
\hline Leblanc and Johnson (2001) & Sputtering & $2 \mathrm{D}$ & UP \\
\hline Leblanc and Johnson (2002) & Sputtering & $1 \mathrm{D}$ & MD \\
\hline $\begin{array}{l}\text { Krestyanikova and Shema- } \\
\text { tovich (2005) }\end{array}$ & DR & 1D/1D & FSDCS/HS-isotropic \\
\hline Cipriani et al. (2007) & DR, sputtering & $1 \mathrm{D} / 1 \mathrm{D}$ & MD-UP-HS \\
\hline Chaufray et al. (2007) & DR, sputtering & 2D/1D, rotation & UP \\
\hline Valeille et al. (2009) & DR & 3D/3D, winds, rotation & HS-isotropic \\
\hline Fox and Hac (2009) & DR & 1D/1D & FSDCS/HS-isotropic \\
\hline
\end{tabular}

To describe Mars' hot oxygen corona, which is produced by $\mathrm{O}_{2}^{+}$dissociative recombination, first a realistic $\mathrm{O}_{2}^{+}$density profile, electron temperature profile, and a neutral density profile (to calculate collisions) are assumed to compute the production rates of hot oxygen. Cipriani et al. (2007), Krestyanikova et al. (2005) and Fox and Hac (2009) assume a spherically symmetry $\mathrm{O}_{2}^{+}$and neutral density profile based on 1D photochemical models (Krasnopolsky 2002; Fox and Hac 2009). To describe sputtering, Leblanc and Johnson (2001) only need neutral density profiles, taken from Zhang et al. (1993), and include a day/night asymmetry in the temperature profile. Hodges (2000) introduces ions and neutral profiles that vary with solar zenith angle, while Chaufray et al. (2007) only uses such variations for $\mathrm{O}_{2}^{+}$ions. Currently, Valeille et al. (2009a) uses the most sophisticated ion and neutral profiles. They use 3D data from the MTGCM model (Bougher et al. 1999), which includes thermospheric winds and planetary rotation. Most of the studies have used the electron temperature profile derived from Viking mission (Hanson and Mantas 1988) except Fox and Hac (2009) who compute the profile. Finally, the erosion of the ionosphere above $\sim 180 \mathrm{~km}$, not included in the MTGCM model or in the photochemical model of Krasnopolsky (2002), should also be taken into account (Fox and Hac 2009).

The easiest way to simulate collisions between hot oxygen and the neutral atmosphere is to use a total cross section and assume an isotropic distribution of scattering angles (hard sphere model). Hodges (2000) and Valeille et al. (2009a) used this approach, and Fox and Hac (2009) tested it. The total cross section used in simulations varies from $1-3 \times 10^{-15} \mathrm{~cm}^{2}$. The isotropic assumption is the main limitation of the hard sphere approach. An accurate description of the $\mathrm{O}-\mathrm{O}$ collisions needs to use 18 separate ground state potential curves for $\mathrm{O}_{2}$ (Tully and Johnson 2001). Kharchenko et al. (2000) derived scattering cross sections between two oxygen atoms by solving the Schrodinger equation of the relative motion of two oxygen atoms in a potential field for different channels corresponding to different states of the $\mathrm{O}_{2}$ molecule. Previously, Hodges (1993) and Shizgal (1999b) used a similar approach to derive scattering cross sections between $\mathrm{O}$ and $\mathrm{H}$ or $\mathrm{D}$ atoms. Kharchenko et al. (2000) found that the statistically averaged (over channels) cross sections are highly peaked at small scattering angles, $\chi$, for energies of few $\mathrm{eV}$. This agrees with the results of Tully and Johnson (2001). They also show that the Landau-Schiff approximation reproduced the statistically averaged (over scattering angles) cross sections as function of 
the energy. Therefore, as pointed out by Fox and Hac (2009), isotropic cross sections overestimate the effect of large scattering angles and therefore the loss of energy for hot atoms (Eq. (3.13)):

$$
\Delta E=E \frac{2 m_{1} m_{2}}{\left(m_{1}+m_{2}\right)^{2}}(1-\cos \chi),
$$

where $m_{1}$ and $m_{2}$ are the masses of the two particles and $E$ is the initial energy of the system.

Using differential cross sections from Kharchenko et al. (2000), Krestyanikova and Shematovich (2005) and Fox and Hac (2009) show that more realistic cross sections could increase the oxygen escape rate by one order of magnitude compared to the isotropic assumption. Another approach used to compute the scattering angles of collisions consists of solving the movement equation of two particles in a potential field. For collisions involving significant energy transfer $(\sim 1 \mathrm{keV})$, the repulsive part of the potential is dominant and a universal potential can be used to describe O collisions (Johnson et al. 2000). This universal potential (Ziegler et al. 1985) is defined by

$$
\begin{aligned}
\Phi(r)= & \frac{Z_{1} Z_{2} q^{2}}{r} \varphi\left(r / a_{u}\right) \\
\varphi(x)= & 0.181 \exp (-3.2 x)+0.5099 \exp (-0.9423 x)+0.2802 \exp (-0.4029 x) \\
& +0.02817 \exp (-0.2016 x)
\end{aligned}
$$

where $Z_{1}$ and $Z_{2}$ are the charge number of the particles ( 8 for oxygen), $q$ the elementary charge, $R$ the distance between the oxygen atoms and $a_{U}=0.8853 a_{0} /\left(Z_{1}^{0.23}+Z_{2}^{0.23}\right)$ where $a_{0}$ is the Bohr radius $(0.529 \mathrm{~nm})$. Knowing, the impact parameter $b$ and the total energy $E$, the scattering angle $\chi$ can be then computed using the usual relation

$$
\chi=\pi-2 b \int_{r_{0}}^{\infty}\left(r^{2}\left(1-\frac{\Phi}{E}\right)-b^{2}\right)^{-1 / 2} \frac{d r}{r},
$$

where $r_{0}$ is the positive root of the equation $r^{2}(1-\Phi / E)-b^{2}=0$.

The impact parameter is derived by

$$
\frac{\pi b^{2}}{\pi b_{\max }^{2}}=\alpha
$$

where $b_{\max }$ is the maximum impact parameter value and $\alpha$ is a random number between 0 and 1 . In the simulations done by Chaufray et al. (2007), $b_{\max }=4 \AA$ \& corresponding to a maximal total cross section $\sim 5 \times 10^{-15} \mathrm{~cm}^{2}$. The universal potential has been used to describe the sputtering of an oxygen atmosphere by $\mathrm{O}^{+}$pick-up ions (Johnson et al. 2000; Leblanc and Johnson 2001; Cipriani et al. 2007; Chaufray et al. 2007). The universal potential has also been used by Chaufray et al. (2007) to describe collisions between hot oxygen (produced by the dissociative recombination of $\mathrm{O}_{2}^{+}$ions) and the ambient atmosphere. However, for energies of few eV, Cipriani et al. (2007) showed that this approach overestimates thermalization compared to differential cross sections calculated from quantum approach (Kharchenko et al. 2000) as used by Krestyanikova and Shematovich (2005).

In Monte Carlo simulations, the environment is divided into several audit cells, where all the macroscopic parameters (velocity distribution function, density, flux, temperature) can be estimated. For 3D approaches (Hodges 2000; Chaufray et al. 2007; Valeille et al. 
2009a), spherical coordinates have been used. In all Monte Carlo simulations, the translational movement is decoupled from intermolecular interactions.

Chaufray et al. (2007) used the algorithm of Bird (1994) to determine the number of collisions in each cell at each time step. In each cell $M$, the maximum number of collisions produced by each individual test particle is

$$
N_{\text {coll }}^{\max }(M)=n(M)(\sigma V)_{\max } d t,
$$

where $n(M)$ is the local density, $(\sigma V)_{\max }$ is an upper value of the product between the cross section and the relative velocity of the test particle and atmospheric particle, and $d t$ the time step of the simulation. Then, for each possible collision, the velocity of the neutral particle is estimated assuming a Maxwellian distribution at the local temperature $T(M)$. The collision is finally taken into account if it satisfies the condition

$$
\frac{\sigma V_{r}}{(\sigma V)_{\max }}<\alpha
$$

where $\alpha$ is a random number between 0 and $1, \sigma$ the cross section of the collision and $V_{r}$ is the relative velocity between the test particle and the atmospheric particle. After a long time simulation the average number of collisions for a given relative velocity $V_{r}$, during $d t$, corresponds to

$$
N_{\text {coll }}(M)=n(M) \sigma V_{r} d t
$$

For each selected collision, the impact parameter is determined from (3.17), and (3.16) and (3.21) are used to derive the angles $(\chi$ and $\varepsilon$ ) that define the post-collisional direction of the two test particles.

$$
\varepsilon=2 \pi \alpha
$$

The most important results given by Monte Carlo simulations are oxygen escape flux and hot oxygen density. Figure 10 shows an example of hot oxygen density, obtained by Chaufray et al. (2007), in the equatorial plane and the velocity distributions at altitudes between 560 and $890 \mathrm{~km}$ for three local times. As expected from the exospheric theory presented above, no satellite, capture or flyby particles are present in the collisionless region. The velocity distribution functions have opposite asymmetries at $6 \mathrm{~h}$ and $18 \mathrm{~h}$ local time. This asymmetry can be explained easily. Most of the hot oxygen atoms are produced at $12 \mathrm{~h}$. These hot atoms come back to the planet in the rotation direction or anti-rotation direction. When they move in the rotation direction $(V t>0)$ they return to the evening hemisphere and when they move in anti-rotation direction $(V t<0)$ they return to the morning hemisphere.

Chaufray et al. (2007) coupled a 3D Monte Carlo model, describing the Martian corona, to a 3D hybrid model, describing the corona's interaction with the solar wind (Modolo et al. 2005). The coupled model was used to compare oxygen escape processes. Escaping fluxes determined by this model are summarized in Table 4. More recently, Valeille et al. (2009b) coupled a 3D MTGCM model to a 3D exospheric model to determine the escape of oxygen due to dissociative recombination for several seasons, solar activity levels and at different epochs in the past. They have shown that oxygen can vary by a factor $\sim 5$ to 7 along with season and solar activity and by a factor $\sim 6$ to 9 historically. These authors also estimate the oxygen escape due to other processes, using simpler assumptions. Valeille et al. (2009b) found that dissociative recombination is the main process of oxygen escape in agreement with Chaufray et al. (2007). However, full coupling between a 3D Martian global circulation model extended to the exobase, a 3D exospheric model and a 3D model describing Mars' 
Fig. 10 Equatorial density and velocity distribution functions for the hot oxygen component obtained at altitudes between 560 and $890 \mathrm{~km}$ at three local times obtained by Chaufray et al. (2007). The red circle and the red hyperbolae on velocity distribution function figures indicate the boundaries for escaping particles and closed trajectories (satellite or flyby see also Fig. 8). The positive sense of the tangential velocity is the direction of the planetary rotation indicated by the blue arrow. More details can be found in Chaufray et al. (2007)

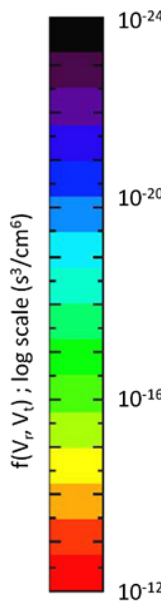

$10^{-24}$

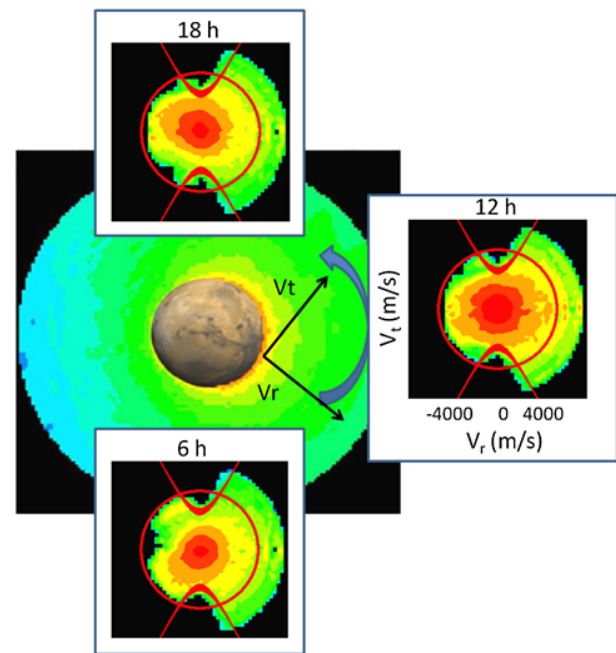

Table 4 Oxygen escape flux estimated by the Monte Carlo simulations of Chaufray et al. (2007) at low and high solar activity for different processes

\begin{tabular}{lll}
\hline Process & $\begin{array}{l}\text { Low } \\
\text { solar activity }\end{array}$ & $\begin{array}{l}\text { High } \\
\text { solar activity }\end{array}$ \\
\hline Dissociative recombination & $1 \times 10^{25}$ & $4 \times 10^{25}$ \\
Pick-up ions escape & $2 \times 10^{23}$ & $3 \times 10^{24}$ \\
ENA escape & $4 \times 10^{22}$ & $4 \times 10^{23}$ \\
Sputtering & $2 \times 10^{23}$ & $7 \times 10^{23}$ \\
\hline
\end{tabular}

interaction with the solar wind is still needed to accurately and self-consistently investigate the evolution of oxygen escape processes. This type of model could determine the amount of water that has escaped to space historically, and in particular in past epochs when the solar wind was likely denser and faster.

\section{Summary}

Numerical simulations help us understand the dynamics of ionized and neutral particles near Venus, Mars, and Titan. The modeling approach that should be used to study plasma and neutral phenomena depends on the relevant scales of the interaction. This paper describes the methodology, advantages, and limitations of several self-consistent plasma models that have been used to model the plasma and neutral dynamics near Venus, Mars, and Titan.

Fully kinetic models, which simulate ions and electrons as particles, were shown to make the fewest assumptions and therefore include the most accurate physics. However, these models must resolve electron inertial scales and include fast electromagnetic waves. Therefore, fully kinetic models are typically used to studying small regions or fast processes. MHD models simulate ions and electrons as fluids. These models include the most assumptions; therefore they often neglect important physical processes, particular in collisionless regions where the fluid assumptions begin to fail and ion cyclotron effects become important. However, the numerical simplicity of MHD models allows them to simulate large regions over long time scales. Hybrid models are an intermediate between fully kinetic models and 
MHD models. In hybrid models, ions are simulated as macroparticles and electrons are simulated as a mass-less fluid. Hybrid simulations can be used to study much larger regions than fully kinetic models and include both phenomena that occur at ion scales and non-thermal ion distributions; however, compared to MHD models they are still somewhat limited in resolution and/or simulation time.

This paper also describes Chamberlain's method and the Monte Carlo method, which are used to describe the dynamics of neutral particles in planetary exospheres. These models are important because they help us understand Martian atmospheric escape both today and historically. Efforts are currently underway to couple various exospheric models to plasma models in order get a complete description of the interaction of the atmosphere's of unmagnetized bodies with the plasma and electromagnetic fields in their space environment.

Acknowledgements The authors acknowledge the support of EUROPLANET RI project (Grant agreement no.: 228319) funded by EU; and also the support of the International Space Science Institute (Bern). RM acknowledges support from the ANR (ANR-09-JCJC-0038 and ANR-09-BLAN-223).

Open Access This article is distributed under the terms of the Creative Commons Attribution Noncommercial License which permits any noncommercial use, distribution, and reproduction in any medium, provided the original author(s) and source are credited.

\section{References}

M.H. Acuña et al., J. Geophys. Res. 106(E10), 23403-23418 (2001)

H. Backes et al., Science 308, 992 (2005). doi:10.1126/science.1109763

S. Barabash et al., Science 315(5811), 501-503 (2007). doi:10.1126/science.1134358

C.A. Barth et al., J. Geophys. Res. 76, 2213 (1971)

R. Bauske et al., J. Geophys. Res. 103(A10), 23635-23638 (1998)

M. Benna et al., Geophys. Res. Lett. 36, L04109 (2009). doi:10.1029/2008GL036718

J.-L. Bertaux, Planet. Space Sci. 26, 431 (1978)

C. Bertucci et al., Geophys. Res. Lett. 30(2), 1099 (2003). doi:10.1029/2002GL015713

C. Bertucci et al., Science 321, 1475 (2008). doi:10.1126/science. 1159780

C. Bertucci et al., Space Sci. Rev. (2011, submitted)

N. Besse et al., Int. J. Appl. Math. Comput. Sci. 17, 361-374 (2007). doi:10.2478/v10006-007-0030-3

G.A. Bird, Molecular Gas Dynamics and the Direct Simulation of Gas Flows (Clarendon, Oxford, 1994)

C.K. Birdsall, A.B. Langdon, Plasma Physics via Computer Simulation (McGraw-Hill, New York, 1985)

A. Boesswetter et al., Ann. Geophys. 22, 4363-4379 (2004)

A. Boesswetter et al., Ann. Geophys. 25, 1851-1864 (2007)

S.W. Bougher et al., J. Geophys. Res. 104, 16591 (1999)

L.H. Brace, A.J. Kliore, Space Sci. Rev. 55(1-4), 81-163 (1991). doi:10.1007/BF00177136

D. Brain et al., Icarus 206, 139-151 (2010)

S.H. Brecht, J.R. Ferrante, J. Geophys. Res. 96(A7), 11209-11220 (1991)

S.H. Brecht, J. Geophys. Res. 102(A3), 4743-4750 (1997)

S.H. Brecht et al., J. Geophys. Res. 105(A6), 13119-13130 (2000)

S.H. Brecht et al., Icarus 206, 164-173 (2010)

S.H. Brecht et al., Earth, Planets and Space. (2011, accepted)

R.T. Brinkman, Planet. Space Sci. 19, 791-794 (1971)

J.W. Chamberlain, Planet. Space Sci. 11, 901 (1963)

J.W. Chamberlain, J. Geophys. Res. 82, 1 (1977)

J.W. Chamberlain, Icarus 44, 651 (1980)

E. Chassefière et al., Planet. Space Sci. 55, 343 (2007)

J.-Y. Chaufray et al., J. Geophys. Res. 112, E09009 (2007). doi:10.1029/2007JE002915

J.-Y. Chaufray et al., J. Geophys. Res. 114, E02006 (2009)

Y. Chen, P.A. Cloutier, J. Geophys. Res. 108, 1382 (2003)

F. Cipriani et al., J. Geophys. Res. 112, E7001 (2007)

A.J. Coates et al., Geophys. Res. Lett. 34, L24S05 (2007). doi:10.1029/2007GL030919

T.E. Cravens et al., Planet. Space Sci. 46, 1193 (1998) 
S.A. Curtis, R.E. Hartle, J. Geophys. Res. 83, 1551 (1978)

E. Dubinin et al., Space Sci. Rev. 206, 209-238 (2006)

E. Dubinin et al., Planet. Space Sci. (2008)

F. Duru et al., J. Geophys. Res. 113, A07302 (2008). doi:10.1029/2008JA0130373

F. Duru et al., Icarus 206(1), 74-82 (2010)

H.J. Fahr, B. Shizgal, Rev. Geophys. Space Phys. 21, 75 (1983)

P.D. Feldman et al., Icarus 214, 394 (2011)

J.L. Fox, A.B. Hac, J. Geophys. Res. 102, 24,005 (1997)

J.L. Fox, A.B. Hac, Icarus 204, 527 (2009)

Y. Futaana et al., Space Sci. Rev. (2011, submitted)

A. Glocer et al., J. Geophys. Res. 114, A12203 (2009). doi:10.1029/2009JA014418

S.L. Guberman, in Physics of Ion-Ion and Electron-Ion Collisions, ed. by F. Brouillard, J.W. McGowan (Plenum, New York, 1983), pp. 167-200

S.L. Guberman, in Dissociative Recombination: Theory Experiment and Applications, ed. by J.B.A. Mitchell, S.L. Guberman (World Scientific, Singapore, 1989), pp. 45-60

J.S. Halekas et al., Geophys. Res. Lett. (2006). doi:10.1029/2006GL026229

W.B. Hanson, G.P. Mantas, J. Geophys. Res. 93, 7538 (1988)

D.S. Harned, J. Comput. Phys. 47, 452-462 (1982)

E. Harnett, R. Winglee, Geophys. Res. Lett. 30 (2003). doi:10.1029/2003GL017852

E. Harnett, R. Winglee, J. Geophys. Res. 110, A07226 (2005). doi:10.1029/2003JA010315

E.M. Harnett, R.M. Winglee, J. Geophys. Res. 111, A09213 (2006). doi:10.1029/2006JA011724

E.M. Harnett, R.M. Winglee, J. Geophys. Res. 112, A05207 (2007). doi:10.1029/2006JA012001

E.M. Harnett, J. Geophys. Res. 114, A01208 (2009). doi:10.1029/2008JA013648

R.E. Hartle et al., J. Geophys. Res. 87, 1383-1394 (1982). doi:10.1029/JA087iA03p01383

R.E. Hartle et al., Geophys. Res. Lett. 33, L08201 (2006). doi:10.1029/2005GL024817

R.R. Hodges, J. Geophys. Res. 78, 8055 (1973)

R.R. Hodges, J. Geophys. Res. 85, 164 (1980)

R.R. Hodges, J. Geophys. Res. 98, 3799 (1993)

R.R. Hodges, J. Geophys. Res. 99, 23229 (1994)

R.R. Hodges, J. Geophys. Res. 104, 8463 (1999)

R.R. Hodges, J. Geophys. Res. 105, 6971 (2000)

R. Jarvinen et al., Adv. Space Res. 41, 1361-1374 (2008)

R.E. Johnson et al., J. Geophys. Res. 105, 1659 (2000)

R.E. Johnson et al., Space Sci. Rev. 139, 355 (2008)

K. Kabin et al., J. Geophys. Res. 104, 2451 (1999)

K. Kabin et al., J. Geophys. Res. 105, 10,761 (2000)

E. Kallio et al., J. Geophys. Res. 103, 4723 (1998)

E. Kallio, P. Janhunen, J. Geophys. Res. 106, 5617-5634 (2001)

E. Kallio et al., Geophys. Res. Lett. 31, L15703 (2004). doi:10.1029/2004GL020344

E. Kallio et al., Planet. Space Sci. 54, 1472-1481 (2006)

E. Kallio et al., Planet. Space Sci. 56, 796-801 (2008)

E. Kallio et al., Icarus 206, 152-163 (2010)

E. Kallio, R. Jarvinen, Earth, Planets and Space (2011, submitted)

J. Kella et al., Science 276, 1530 (1997)

V. Kharchenko et al., J. Geophys. Res. 105, 24,899 (2000)

A. Kidder et al., J. Geophys. Res. 113, A02205 (2009). doi:10.1029/2008JA013100.

J. Kim et al., J. Geophys. Res. 103, 29339 (1998)

V.A. Krasnopolsky, Icarus 107, 5128 (2002)

V.A. Krasnopolsky, Icarus 207, 638 (2010)

M.A. Krestyanikova, V.I. Shematovitch, Sol. Syst. Res. 39, 22 (2005)

A.B. Langdon, Part. Accel. 19, 223-225 (1986)

F. Leblanc, R.E. Johnson, Planet. Space Sci. 49, 645 (2001)

F. Leblanc, R.E. Johnson, J. Geophys. Res 107 (2002). doi:10.1029/2000JE001473

F. Leblanc et al., J. Geophys.Res. 111, E09S11 (2006). doi:10.1029/2005JE002664

S.A. Ledvina, T.E. Cravens, Planet. Space Sci. 46, 1175 (1998)

S.A. Ledvina et al., J. Geophys. Res. 110, A06211 (2005). doi:10.1029/2004JA010771

S.A. Ledvina et al., Space Sci. Rev. (2008). doi:10.1007/s11214-008-9384-6

S.A. Ledvina et al., Earth Planet. Sci. (2011, submitted)

H.I.M. Lichtenegger et al., Space Sci. Rev. 126, 469 (2006)

H.I.M. Lichtenegger et al., Geophys. Res. Lett. 36, L10204 (2009)

Y.J. Liu et al., Geophys. Res. Lett. 26(17), 2689-2692 (1999) 
Y.J. Liu et al., Adv. Space Res. 27, 1837 (2001)

J.G. Luhmann, J. Geophys. Res. 101(E12), 29387-29393 (1996)

J.G. Luhmann, T.E. Cravens, Space Sci. Rev. 55, 1 (1991). doi:10.1007/BF00177138

R. Lundin et al., Geophys. Res. Lett. 17, 877 (1990)

R. Lundin et al., Science 305, 1933-1936 (2004)

R. Lundin et al., Science 311(5763), 980-983 (2006). doi:10.1126/science.1122071

Y.J. Ma et al., J. Geophys. Res. 10, A10 (2002). doi:10.1029/2002JA009293

Y.J. Ma et al., J. Geophys. Res. 109, A07211 (2004). doi:10.1029/2003JA010367

Y. Ma et al., J. Geophys. Res. 111, A05207 (2006). doi:10.1029/2005JA011481

Y.J. Ma et al., Geophys. Res. Lett. (2007). doi:10.1029/2007GL031627

Y.-J. Ma et al., Space Sci Rev. 139(1-4) (2008). doi:10.1007/s11214-008-9389-1

Y.J. Ma et al., J. Geophys. Res. 114, A03204 (2009). doi:10.1029/2008JA013676

C. Mazelle et al., Space Sci. Rev. 111, 115-181 (2004)

A. Matthews, J. Comput. Phys. 112, 102-116 (1994)

M.B. McElroy, Science 175, 443-445 (1972)

M.B. McElroy et al., Science 215, 1614 (1982)

F.J. Mehr, M.A. Biondi, Phys. Rev. 181, 264 (1969)

D.L. Mitchell et al., J. Geophys. Res. 106, 23419 (2001)

R. Modolo et al., Ann. Geophys. 23, 433-444 (2005)

R. Modolo et al., Ann. Geophys. 24, 3403-3410 (2006)

R. Modolo et al., Geophys. Res. Lett. 34, L24S07 (2007). doi:10.1029/2007GL030489

R. Modolo, G.M. Chanteur, J. Geophys. Res. 113, A01317 (2008). doi:10.1029/2007JA012453

K. Moore et al., J. Geophys. Res. 96, 7779-7791 (1991)

K. Murawski, R.S. Steinolfson, J. Geophys. Res. 101, 2547 (1996)

A.F. Nagy, T.E. Cravens, Geophys. Res. Lett. 15, 433 (1988)

A.F. Nagy et al., Ann. Geophys. 8, 251 (1990)

A.F. Nagy et al., J. Geophys. Res. 106(A4), 6151-6160 (2001)

A.F. Nagy et al., Space Sci. Rev. 111, 1 (2004). doi:10.1023/B:SPAC.0000032718.47512.92

D. Najib et al., J. Geophys. Res. 116, A05204 (2011). doi:10.1029/2010JA016272

F. Neubauer et al., J. Geophys. Res. 111, 10220 (2006)

R. Peverall et al., J. Chem. Phys. 114, 6679 (2001)

V. Pohjola, E. Kallio, Ann. Geophys. 28, 743-751 (2010)

J.M. Rodriguez et al., Planet. Space Sci. 32, 1235 (1984)

R.W. Schunk, A.F. Nagy, Ionospheres: Physics, Plasma Physics, and Chemistry (Cambridge University Press, New York, 2009)

V.I. Shematovich et al., J. Geophys. Res. 99, 23,217 (1994)

H. Shimazu, Earth Planets Space 51, 383-393 (1999)

B. Shizgal, Adv. Space Sci. 7, 73 (1987)

B. Shizgal, J. Geophys. Res. 107, 14833 (1999a)

B. Shizgal, Planet. Space Sci. 47, 163 (1999b)

B.D. Shizgal, R. Blackmore, Planet. Space Sci. 34, 279 (1986)

I. Sillanpää et al., J. Geophys. Res. 112, A12205 (2007). doi:10.1029/2007JA012348

S. Simon et al., Ann. Geophys. 24, 1113-1135 (2006)

S. Simon, A. Boesswetter, T. Bagdonat, U. Motschmann, J. Schuele, Ann. Geophys. 25, 117-144 (2007) doi:10.5194/angeo-25-117-2007

S. Simon et al., Planet. Space Sci. 57, 2001-2015 (2009)

G.R. Smith et al., J. Geophys. Res. 83, 3783 (1978)

D. Snowden et al., J. Geophys. Res. 112, A1221 (2007). doi:10.1029/2007/JA012393

A.I. Stewart et al., J. Geophys. Res. 97, 91 (1992)

D.J. Strickland et al., J. Geophys. Res. 77, 4052 (1972)

D.J. Strickland et al., J. Geophys. Res. 78, 4547 (1973)

T. Tanaka, J. Geophys. Res. 98, 17251-17262 (1993)

T. Tanaka, K. Murawski, J. Geophys. Res. 102, 19,805 (1997). doi:10.1029/97JA01474

N. Terada et al., J. Geophys. Res. 114, A09208 (2009). doi:10.1029/2008JA013937

G. Tóth et al., J. Comput. Phys. 227, 6967-6984 (2008)

C. Tully, R.E. Johnson, Planet. Space Sci. 49, 533 (2001)

T. Umeda et al., Comput. Phys. Commun. 156(1), 73-85 (2003). doi:10.1016/S0010-4655(03)00437-5

T. Umeda, J. Geophys. Res. 115, A01204 (2010). doi:10.1029/2009JA014643

A. Valeille et al., J. Geophys. Res. 114, E11005 (2009a)

A. Valeille et al., J. Geophys. Res. 114, E11006 (2009b)

S. Vennerstrom et al., Geophys. Res. Lett. 30, 1369 (2003) 
A. Vidal-Madjar, J.-L. Bertaux, Planet. Space Sci. 20, 1147 (1972)

D. Vignes et al., Geophys. Res. Lett. 27, 49 (2000)

J. Villasenor, O. Buneman, Comput. Phys. Commun. 69, 306 (1992)

J.H. Waite et al., Science 308(5724), 982-986 (2005)

J.E. Wahlund et al., Science 308, 986-989 (2005)

H.Y. Wei et al., Geophys. Res. Lett. 34, L24S06 (2007). doi:10.1029/2007GL030701

R.M. Winglee et al., J. Geophys. Res. 114, A05215 (2009). doi:10.1029/2008JA013343

M.H.G. Zhang et al., J. Geophys. Res. 98, 10,915 (1993)

T.L. Zhang et al., Geophys. Res. Lett. 37, L14202 (2010). doi:10.1029/2010GL044020

J.F. Ziegler et al., The Stopping and Ranges of Ions in Matter (Pergamon, New York, 1985) 